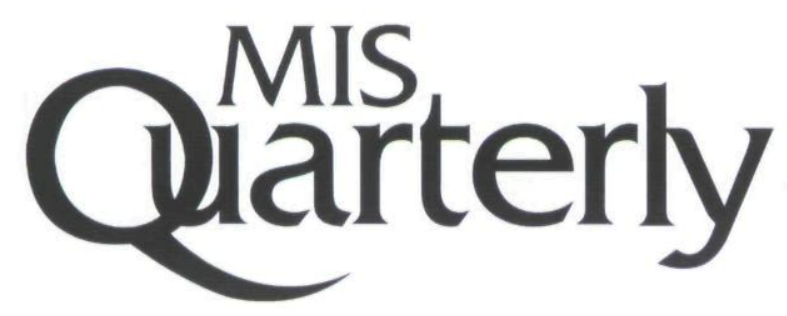

SPECIAL ISSUE

\title{
Assessing Value in ORganizational KNOWLEDGE CREATION: Considerations FOR KNOWLEDGE WORKERS ${ }^{1}$
}

By: Andrew N. K. Chen

Department of Information Systems

Arizona State University

Mail Code 4606

Tempe, AZ 85287-4606

U.S.A.

andrew.chen@asu.edu

Theresa M. Edgington

Department of Information Systems

Arizona State University

Mail Code 4606

Tempe, AZ 85287-4606

U.S.A.

theresa.edgington@asu.edu

\section{Abstract}

To maintain competitive advantage, a firm's investment decisions related to knowledge creation are likely to be strategic in nature. However, strategic investments usually have an element of risk linked to uncertain and deferred investment benefits. To date, such investment decisions relating to knowledge workers have not been exten-

\footnotetext{
${ }^{1} \mathrm{~V}$. Sambamurthy and Mani Subramani were the accepting senior editors for this paper.
}

sively researched. In this paper, we explore the following research question: How do we strategically assess knowledge creation over time giving consideration to complex decision criteria in order to improve organizational value? We develop a model based on economic and organization theory for assessing organizational value with regard to knowledge creation investments. Our model prototype provides managers with a learning tool relating to the timing and selection of knowledge creation investments. Our own use of the tool in simulation experiments yielded several insights which suggest that the decisions typically made by managers may dilute knowledge creation investments. Our results demonstrate that the organizational benefit of knowledge creation processes should be well aligned with near-term tasks. Under instances of high knowledge depreciation, however, it is unlikely that individual workers can optimize knowledge creation process decisions without organizational involvement in matching skills to task complexities. The organizational benefits of consistent and frequent knowledge creation process participation increase over time as the match of skills and task complexities improve.

Keywords: Knowledge management, knowledge creation, organizational dynamics, task characteristics, organizational theory, economic theory, simulation 


\section{Introduction}

Knowledge management has emerged as an important research topic for information systems and management researchers since the 1990s (e.g., Alavi and Leidner 2001; Davenport and Grover 2001; Hahn and Subramani 2000; Nonaka 1991,1994). While knowledge creation is fundamental to the survival of a business, it has not been extensively researched beyond organizational theory. It would be easy (yet incorrect) to surmise that many firms have developed sophisticated, formal strategies within the context of organizational knowledge creation. Many managers do appear to consider training and other knowledge development programs during their operational budgeting tasks. Ironically, these programs are often loosely constructed and quickly cut during economic shortfalls. In this study, we emphasize and investigate the importance of active governance of strategic knowledge creation for organizations seeking sustainable competitive advantage.

Alavi and Leidner (2001) consider the firm's ability to create knowledge and to take action upon it as the basis for competitive advantage; however, knowledge creation (KC) comes with a price. A substantial KC challenge lies in the investment tradeoff between future benefits and current tasks, ${ }^{2}$ balancing essential near-term goals without compromising long-term competitiveness. Opportunities for organizational synergy can also influence how $\mathrm{KC}$ process ${ }^{3}$ investment decisions are made. $\mathrm{KC}$ process decisions have an impact on innovation and strategic management. In this paper, we explore the following research question: How do we strategically assess knowledge creation over time giving consideration to complex

\footnotetext{
${ }^{2}$ Task: An assigned piece of work to be finished within a specified time. Thus, task is a directed effort performed by the worker with a measurable result that benefits the organization.
}

${ }^{3} \mathrm{KC}$ process: A unit of activity, either instructed or selfadministered, undertaken for the purpose of improving upon individual or organizational tasks, with respect to quality or efficiency. Thus, a KC process is an assigned or unassigned activity with future tasks for which an increased benefit is expected (Becker 1977; Mincer 1962). decision criteria in order to improve organizational value?

As markets continue to aggressively evolve and while knowledge creation processes typically involve some monetary and opportunity investment, we find that the decision-making criteria needed by managers to evaluate knowledge creation processes for their knowledge workers are highly complex and, therefore, should be carefully managed. Our research contributes to the literature by considering how cross-discipline theories interact to influence managers' KC process investment decisions. We develop an analytical model based on these theoretical motivations by combining a complex set of decision criteria for assessing the value of strategic, organization-level knowledge creation. This model allows for expansion and refinement by understanding each organization's specific situation including the characteristics of the organization's knowledge workers, $\mathrm{KC}$ process characteristics and investment options, the costs associated with the work tasks, and the timing considerations. Using simulation analysis, we reveal results that are contrary to typical management practice along with those that confirm typical practice. Hypotheses are presented and tested statistically with simulation results. Implications to both researchers and practitioners are provided.

Our results demonstrate that the organizational benefit of knowledge creation processes should be well aligned with near-term tasks. Under instances of high knowledge depreciation, however, it is unlikely that knowledge creation process choices made individually by workers would result in maximizing corporate value without organizational involvement in matching skills to task complexities. The organizational benefits of consistent and frequent knowledge creation process participation increase over time as the match between skills and task complexities improve.

\section{Problem Elaboration}

Within today's knowledge economy where knowledge work exceeds the production of capital goods as evidenced by the United States gross national product, an organization competes through unique 
capabilities and leadership to sustain some differentiation from competing organizations. For many knowledge workers, external sources of new knowledge creation must be regularly obtained or the worker is at risk of obsolescence (i.e., knowledge creation becomes an ongoing requirement). It is seldom accurate to assume that knowledge workers (unlike their production-oriented counterparts) become, and stay, proficient solely from repetition and reinforcement of their past actions. Knowledge worker tasks include (1) those tasks similar to traditional production where on-the-job performance includes task repetition and (2) those tasks where scanning for new information or knowledge inside or outside the organization leads to knowledge creation. It is this second set, which differentiates knowledge workers' tasks from those of traditional production-oriented workers, upon which we focus this study.

Knowledge worker tasks appear highly sensitive to change and change is exacerbated by the Internet and associated technology. As a result, ongoing knowledge creation becomes an adaptation defense. Consider the role of the enterprisesoftware developer. Viewed traditionally, by continuing to program in the same programming language, the worker reinforces and gains sufficient skills to eventually be qualified as an expert (i.e., repetitive reinforcement provides the basis for expertise). However, this strategy has not worked for COBOL developers, whose marketability (a relatively unbiased value estimator of skills) has not been enhanced by on-the-job reinforcement, and may even have been compromised by it (Dice Salary Survey $2004^{4}$ ). To be considered for new, competitive initiatives, experienced developers need to become proficient in an advanced language, such as Java or C\#, with proficiency at the level of J2EE or .NET compliance for programming. Continuous, externally obtained, ${ }^{5}$

\footnotetext{
${ }^{4}$ Dice Salary Survey: http://marketing.dice.com/ rateresults/index.asp; accessed February 12, 2004.

${ }^{5}$ External KC occurs when resources beyond the repetitive activities are required to perpetuate or optimize the continued level of achievement for the worker or for the organization. External KC processes may range from self-training (e.g., reading manuals and journals) to structured training courses. Training is a typical, but
}

technological KC is not only anticipated, but expected.

The process in which this knowledge creation occurs may be informal, formal, or structured. Informal KC lacks an organizational sponsor, occurring through proximity or from specific interest. Whether sitting in adjoining cubicles or participating in informal communities of practice (Wenger and Snyder 2001), individuals may learn as much via informal channels as by other venues. However, individual benefit does not automatically result in organizational benefit unless specific attention beyond that motivated by self-interest is given to achieving organizational goals.

The organization has more control over formal or structured KC processes, which are the focus of our model. Formal KC processes have an organizational sponsor and an intentional objective. Formal KC processes, which often are manifested in organized meetings (face-to-face or electronic), can be distinguished from structured $\mathrm{KC}$ processes. Structured KC processes, such as training programs, subsume formal $\mathrm{KC}$ processes, but also include a specified (and, generally, dedicated) timeframe, an agenda, a sequence of defined events, and a clear leader (facilitator or instructor). Both formal and structured $\mathrm{KC}$ processes, to be effective, should include both repetition and feedback. Repetition is a requirement in learning theory (Piaget 1969), and feedback implies a presentation and interpretation of results from the participants to the organizational management. Thus, formal and structured KC processes exhibit a systematic orientation.

The knowledge worker can assume the $\mathrm{KC}$ burden and pursue learning solely from external sources and observations, in which case the efficiency of learning, the type of learning process (efficiencyoriented or innovative), the timing of it (i.e., during slack time at work or on one's own time), and the organizational value of such learning are solely the decision of the worker. Individuals may select

notexclusive, form of knowledge creation; other KC activities include task teams, ad hoc committees, or formal product innovation activities. 


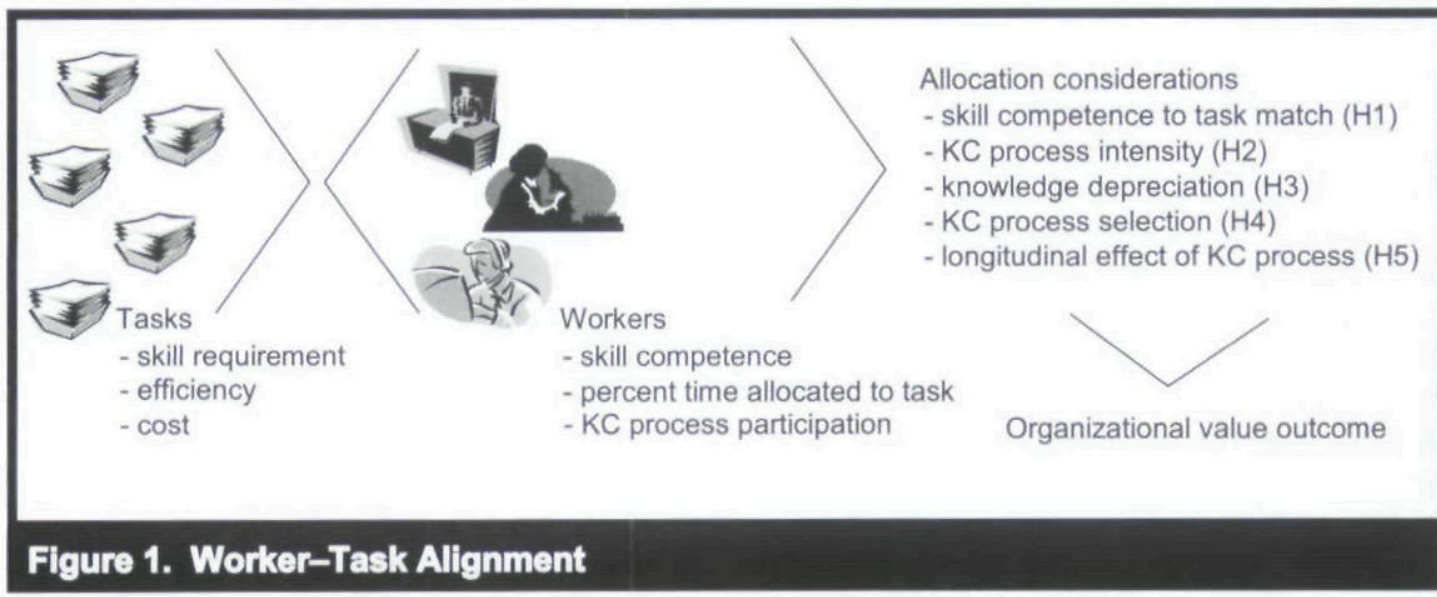

knowledge creation activities that maximize personal utility but not organizational utility. For instance, a knowledge worker finishing a task ahead of schedule has three selection choices: (1) participate in a KC process benefitting the organization, (2) work on another task benefitting the organization, or (3) choose an individual process of purely personal utility, such as monitoring one's stock portfolio or relieving stress by playing an online game.

Alternatively, the organization can assume some responsibility to align learning in such a way that knowledge creation for the organization is optimally achieved with minimal impact taking into account both current and long-term organizational tasks. The individual generally determines selection and participation in informal KC processes, and agency theory would suggest that individual self-interest drives these decisions, resulting in at least a modest if not substantial inefficiency to the organization. As such, our model considers a number of decision criteria pertinent to the organizational value of formal and structured $\mathrm{KC}$ processes.

The manager of an organization has the opportunity and responsibility to strategically align knowledge workers' assignments to tasks or KC processes (Figure 1). An organization has a specific set of knowledge tasks that are required to meet corporate goals competitively. A set of workers exists, or will be hired, to execute these tasks. Each task has a certain skill requirement which characterizes the task in terms of complexity and difficulty. Resources, such as technology, which are available to any worker performing the task, are included in the complexity determination. The required skill to complete a task is specific to the task. The corresponding cost of performing a task in a time period is also specific to the task, but is influenced by the worker(s) assigned to the task. Each worker possesses a competency level for each specific task and can be assigned a percentage of time to devote to that task. A worker can be given one or more tasks during a time period, which is determined by the fit between the task's skill requirement and the worker's competence for the task(s). When the organization's tasks are aligned with the workers' competencies, an efficient allocation state exists. Organizational goals are attained; the organization's competitiveness is strengthened; and workers can be expected to be satisfied with a task contribution that matches their competencies.

New skills can be obtained by knowledge workers by participating in knowledge creation processes. However, these processes may have direct costs and can be expected to have opportunity costs when they remove a knowledge worker from performing a desired task. The opportunities for improved organizational value introduce allocation decisions and considerations which the manager can consider; these allocation decisions are summarized in Figure 1 and correspond to hypotheses which are more fully described in the hypotheses development section of this paper. 


\section{Literature Review and Conceptual Model}

From a business domain perspective, "one of the major challenges for management is to understand the role of knowledge and learning for organizational change and business success" (Pawlowsky 2001). We consider the value of knowledge delivery to be essential within an organizational context; therefore, we focus on the task as the central activity of knowledge workers.

The manager's decision criteria, with regard to KC process investments, are complex and not adequately addressed by any one theory. Issues of competitive outcome and market satisfaction of organizational goals must intertwine with the interaction between manager and individual, in addition to the potential leverage afforded by organizational synergy. We find (see Table 1) several theories that influence each of these perspectives, which we coalesce into our developed model. We start with the resource-based view (RBV) of the firm, incorporate various cognitive elements (learning theory, cognitive decay, organizational memory, and absorptive capacity), and acknowledge various economic theories (complementarity, human capital theory, transaction cost theory, and agency theory). These constructs are explained in the following sections along with additional research which has influenced their development and integration into our model.

In our model, we maximize the sum of benefits ( $\Pi$ $\left.=\sum_{t=1}^{T} \pi_{t}\right)$ of an organization over all time periods under consideration where

$$
\pi_{t}=R_{t}-C_{t}^{\text {fixed }}-C_{t}^{\text {variable }}-C_{t}^{\text {training }}-C_{t}^{\text {opportunity }}
$$

Furthermore, we incorporate the net present value (NPV) as $\sum_{t=1}^{T} N P V\left(\pi_{t}\right)$ to capture the discounting of these benefits over time. We define the revenues $(R)$ of an organization as the performance on tasks based on workers' contributions. While workers participate in KC processes and improve their competence, the organization benefits from higher efficiency and output task performance. Four types of costs are considered for deriving the organization's value. We classify the compensation that a worker receives from an organization into two different types: skill-oriented (fixed compensation) and task-oriented (variable compensation). Fixed compensation $\left(C^{f i x e d}\right)$ is based on the market value of a worker's demonstrated competence at the beginning of a time period $t$, and variable compensation $\left(C^{\text {variable }}\right)$ for a worker is based on the contribution of the worker toward successfully completing task(s) during the time period $t$. The cost of participating in KC processes $\left(C^{\text {training }}\right)$ is also considered. Finally, opportunity cost in a time period is the potential lost benefit from that portion of time in a specific time period where workers participate in $\mathrm{KC}$ processes but could have contributed to performing tasks.

The scenario in which our model applies considers a broadly applicable management decision: knowledge worker assignment to requisite work tasks and $\mathrm{KC}$ processes. At the beginning of a time period $(t)$, an organization has knowledge workers $(i=1, \ldots, l)$ and organizational tasks $(j=1$, ..., J). In a typical real-world environment, an organization has a finite set of knowledge workers and a set of required and desired tasks that often exceed the capabilities of these workers in any one time period. The organization can improve upon the ability to respond to these tasks by improving upon the skills of the workers through knowledge creation processes.

During a time period, individuals may perform tasks, such as developing a program and responding to project e-mail, and they may participate in $\mathrm{KC}$ processes, such as logging onto an e-learning course for a new technology or corporate initiative. Our general model allows a worker $i$ during a non-discrete time period to perform more than one task. Our general model also permits any worker $i$ during a time period to participate in more than one KC process. We introduce a variable $\left(X_{i j t}\right)$ to indicate the portion of worker is time devoted to performing task $j$ during time period $t$, and a variable $\left(Y_{i j t}\right)$ to indicate the 


\begin{tabular}{|c|c|c|}
\hline $\begin{array}{l}\text { Organizational } \\
\text { Perspective }\end{array}$ & Model Construct(s) & Theory and Supporting Research \\
\hline $\begin{array}{l}\text { Competence and } \\
\text { Increased } \\
\text { Knowledge }\end{array}$ & $\begin{array}{l}\text { Demonstrated competence of a } \\
\text { worker }(\varepsilon) \\
\text { Actual or potential gain in } \\
\text { competence }(G) \text { due to } \mathrm{KC}\end{array}$ & $\begin{array}{l}\text { Resource-based view of the firm (Barney } \\
\text { 1991; Hitt et al. 1998; Ndlela and Toit } \\
\text { 2001) } \\
\text { Human capital theory (Becker 1962) }\end{array}$ \\
\hline Intensity & $\mathrm{KC}$ process intensity $(v)$ & $\begin{array}{l}\text { Learning Theory (Anderson 1995; Schilling } \\
\text { et al. 2003) } \\
\text { Absorptive Capacity (Cohen and Leventhal } \\
\text { 1990) }\end{array}$ \\
\hline $\begin{array}{l}\text { Knowledge } \\
\text { Depreciation }\end{array}$ & $\begin{array}{l}\text { Depreciation rate for a specified } \\
\text { time period }(\delta) \text { due to either } \\
\text { decay or obsolescence }\end{array}$ & $\begin{array}{l}\text { Cognitive decay (Schacter 2001) } \\
\text { Knowledge obsolescence (Flamhotltz and } \\
\text { Lacey 1981; Nonaka and Takeuchi 1995; } \\
\text { Zell 2001) } \\
\text { Organizational Memory (Alavi and Leidner } \\
\text { 2001; Argote et al. 1990; Darr et al. 1995) }\end{array}$ \\
\hline $\begin{array}{l}\text { Employee } \\
\text { Contracts }\end{array}$ & $\begin{array}{l}\text { Market-determined compensation } \\
\quad\left(C^{f i x e d}\right) \\
\text { Task completion compensation } \\
\quad\left(C^{\text {variable }}\right)\end{array}$ & $\begin{array}{l}\text { Transaction cost theory (Coase 1937; Simon } \\
\text { 1951; Williamson 1980) } \\
\text { Agency theory (Jensen and Meckling 1976) }\end{array}$ \\
\hline $\begin{array}{l}\text { Organizational } \\
\text { Synergy }\end{array}$ & $\begin{array}{l}\text { Underlying factor considering the } \\
\text { effects of eliminating duplicate } \\
\text { work tasks and aligning worker } \\
\text { competencies to tasks }\left(M^{\top}\right)\end{array}$ & $\begin{array}{l}\text { Complementarity (Barua 1996; Edgeworth } \\
\text { 1881; Laursen and Foss 2003; Milgrom } \\
\text { and Roberts 1995; Prat 2002) }\end{array}$ \\
\hline $\begin{array}{l}\text { Specific KC } \\
\text { Costs }\end{array}$ & $\begin{array}{l}\text { Direct costs: fees/tuition, travel } \\
\text { and living expense, required } \\
\text { materials }\left(C^{\text {training }}\right)\end{array}$ & $\begin{array}{l}\text { Human capital theory (Becker 1962; Josefek } \\
\text { and Kauffman 1999; Malcolmson et al. } \\
\text { 2003) }\end{array}$ \\
\hline $\begin{array}{l}\text { Opportunity } \\
\text { Costs }\end{array}$ & $\begin{array}{l}\text { Lost performance due to } \mathrm{KC} \\
\left.\text { participation ( } C^{\text {opportunity }}\right)\end{array}$ & $\begin{array}{l}\text { Transaction cost theory (Baland and } \\
\text { Francois 2000; Coase 1937; Hubbard } \\
1995 \text { Stevens 1994; Vera-Munoz 1998) } \\
\text { Human capital theory (Booth et al. 2002; } \\
\text { Shaw 1992) }\end{array}$ \\
\hline
\end{tabular}

portion of worker i's time devoted to participating in a KC process for task $j$ during time period $t$, where $X_{i j t}, Y_{i j t} \in[0,1] ; \forall i=1, \ldots, l$ and $\forall j=1, \ldots, J$. During a time period, knowledge workers may perform tasks, participate in KC processes in order to increase their competence for future tasks, or do both. Therefore, a worker's time consumed during any time period $t$ will be the sum of the time in performing these tasks and the time toward participating in KC processes. Therefore, $\sum_{j=1}^{j} X_{y t}+\sum_{j=1}^{J} Y_{y t}=1$ for any worker in any time period. 


\section{Competence and Increased Knowledge}

Both management theory and human capital economics acknowledge the role of worker competence as a critical resource for organizations. The resource-based view (RBV) (Barney 1991) considers workers as valuable organizational resources. Human capital theory (HCT) (Becker 1962) explains differences in income and productivity in terms of ability (time to reach a desired skill level) and opportunity (career entry economic and social conditions). From the context of RBV, managers consider knowledge workers as competent organizational resources from which competitive advantage can be obtained within a particular market. RBV provides a foundational motivation toward the continued development of skills within the organization's human resource. Competitive advantage is maintained and sustained through a rare, inimitable, and valuable resource mix. We consider that organizations exist in a knowledge economy, characterized by aggressive, evolutionary, and dynamic market conditions. Sustained competitive advantage depends heavily on human knowledge and the ability to learn and adapt in unique ways. In a study by Christianse and Venkatraman (2002), $\mathrm{RBV}$ was found to be superior to transaction cost economics with regard to the creation of human competence.

Competence relates to the ability to complete a task satisfactorily within a specified time period. On any particular task, workers are likely to differ in their competence, ranging from high competence to low competence even when other resources are equally available. Increasing a worker's knowledge of a task increases the competence level. The continuous quest for new knowledge feeds directly into achieving and sustaining competitive advantage (Hitt et al. 1998; Ndlela and Toit 2001).

In a strategic sense, increased knowledge can relate to the introduction of a novel manifestation resulting in an innovation. A positive relationship has been found between innovation and knowledge acquisition within an organizational context
(Child and Heavens 2001). It is interesting to note that technology introduction does not dampen an organization's innovative ability (Purvis et al. 2001) presuming that workers are adequately trained with regard to such technology. In an operational sense, increased knowledge can result in improved worker competence and task performance quality, a production efficiency function.

For our model, at the beginning of each time period, we assume that a firm has an objective mechanism to measure each worker's demonstrated competence (i.e., a firm does not consider any actual or potential competence that the worker may possess but has not yet demonstrated.). We use $\varepsilon_{i j t}$ to represent the demonstrated competence of worker $i$ for performing task $j$ at the beginning of time period $t$ and $\varepsilon_{i p} \in[0,1] ; \forall i=1, \ldots, I$ and $\forall j=$ $1, \ldots, J$.

\section{Intensity}

An element of variability, which we call intensity, occurs with respect to the $\mathrm{KC}$ process, to the learning that occurs within the learner, and to the task itself. These intensities are critical to adequately derive an understanding of value within any formal $\mathrm{KC}$ process.

The intensity with respect to a $\mathrm{KC}$ process relates to the sophistication of the knowledge intended for the participant. Academic courses are a useful example of $\mathrm{KC}$ processes of differing intensity. Courses at the 100-level (i.e., for freshmen) are considered to be less intense than a 700-level (i.e., for Ph.D. students) course. For practitioners, training courses may be categorized as introductory (basic) or advanced. The amount of intended knowledge during a $\mathrm{KC}$ process may characterize intensity; i.e., one will work harder during this $\mathrm{KC}$ process than one would in a less intense $\mathrm{KC}$ process, and the level of sophistication of the KC process allows for a substantial increase in the competence of the knowledge worker. Intensity of a $\mathrm{KC}$ process may also relate to the amount of detail covered. A low intensity $\mathrm{KC}$ process for a knowledge worker may merely provide an overview of objectives, rationale, and procedural tips; 
however, the process can become more intense by adding exercises and role playing to further reinforce the objectives and procedures. The intensity of the $\mathrm{KC}$ process with respect to knowledge worker competence (relating to the topic area) is considered when assigning workers to $\mathrm{KC}$ processes; less intense KC processes are typically better suited for new or less-competent workers.

The second intensity type relates to the worker's absorptive capacity or learning rate. Workers leave meetings, courses, and other $\mathrm{KC}$ processes with different levels of new knowledge. Learning is influenced by unique, individual mental models (Anderson 1995) such that no two individuals obtain the same knowledge from any $\mathrm{KC}$ process. Groups working on different yet similar types of problems over time (related variation) learn at significantly faster rates than do teams working under specialization (repetition, no variation) or unrelated variation (Schilling et al. 2003) by deepening cognitive structure or stimulating insightful synthesis between different problem domains.

A knowledge task possesses an intensity level. Some tasks are more difficult than others no matter who performs the task. Additionally, task completion and quality will be affected by the competence of the worker assigned to it. Worker task assignments can improve in value for the firm if productivity from completing a task is increased (efficiency) or if the intensity of the task a worker does perform is increased (i.e., replaced by a higher value task). $\mathrm{KC}$ processes can increase the worker's competence and as a consequence either increase efficiency of performing the existing task (providing slack for additional work) or qualify the worker for a high-intensity task.

In summary, a specific KC process is directed toward enhancing the knowledge and competence of performing a specific task. We consider that KC processes can differ with respect to intensity. We assign a value between 0 and 1 for intensity of a $\mathrm{KC}$ process for a specific task; i.e., $v_{j} \in[0,1] ; \forall j$ $=1, \ldots, J$ (tasks). For our model, the increased competence $\left(G_{i j t}\right)$ for worker $i$ by participating in a $\mathrm{KC}$ process $j$ during time period $t$ will depend on the intensity of the $\mathrm{KC}$ process $\left(v_{j}\right)$, the portion of time invested in the $\mathrm{KC}$ process $\left(Y_{i j t}\right)$, the existing competence for task $j\left(\varepsilon_{i j t}\right)$, and a noise term $(\rho)$. Therefore,

$$
G_{i j t}=v_{j} \times f\left(Y_{i j t}\right) \times O\left(\varepsilon_{i j t}\right)+\rho
$$

A strictly increasing S-curve function, $f(\cdot)$, considers both learning and saturation effects of the time devoted to a KC process. That is, the learning effect will gradually increase and then saturate at 100 percent as the amount of time devoted $\left(Y_{i j t}\right)$ increases. Therefore,

$$
\begin{gathered}
f\left(Y_{i j t}\right)=\frac{1}{1+\alpha e^{-\beta Y_{j t}}} \\
\text { where } \alpha>1 \text { and } \beta>0
\end{gathered}
$$

When no time is devoted for learning, there will be no competence gain for a worker; i.e., $f(0)=0$. Therefore, to ensure this condition, we modify the formulation as

$$
\begin{aligned}
f\left(Y_{i j t}\right)= & \left(\frac{1}{1+\alpha e^{-\beta Y_{j t}}}-\frac{1}{1+\alpha}\right) \times\left(\frac{1+\alpha}{\alpha}\right) \\
& \text { where } \alpha>1 \text { and } \beta>0
\end{aligned}
$$

$O(\cdot)$ is also an S-curve function. It is argued that participants of a KC process will gain differently depending on their existing competencies and the nature of the KC process (basic or advanced). For example, with a novice and expert user of data mining software in the same introductory class, the novice's skill level should increase significantly while the expert user's skill level may be only moderately improved, if at all. However, in an advanced class, the expert's skill level should improve while the novice could be expected to be overwhelmed with only a minimal improvement, if any at all. This reinforces the need for planning with respect to $\mathrm{KC}$ process investments. We introduce $O_{b}(\cdot)$ as an S-curve function with a strictly decreasing trend. It is argued that by participating in an introductory KC process, the greater the current competence $\left(\varepsilon_{i j t}\right)$ of a worker, the less the increase of the worker's competence (a limited learning result). Therefore, 


$$
O_{b}\left(\varepsilon_{i j t}\right)=\frac{1}{1+\alpha^{\prime} e^{-\beta^{\prime} \varepsilon_{i t}}}
$$

$$
\text { where } \alpha^{\prime}>0 \text { and } \beta^{\prime}<0
$$

We also introduce $O_{a}(\cdot)$ as an S-curve function with a strictly increasing trend. It is argued that by participating in an advanced $\mathrm{KC}$ process, the greater the current competence that a worker has, the greater the worker's competence increases (with a better learning result). Therefore,

$$
O_{a}\left(\varepsilon_{i j t}\right)=\frac{1}{1+\alpha^{n} e^{-\beta^{n} \varepsilon_{i j t}}}
$$

$$
\text { where } \alpha^{\prime \prime}>1 \text { and } \beta^{\prime \prime}>0
$$

\section{Knowledge Depreciation}

The concept of knowledge depreciation with respect to competitive position is important when considering an organization's strategic needs in the context of aggressive and evolving markets. Knowledge depreciation may occur either from (1) knowledge decay or (2) knowledge obsolescence. Empirical research supports the notion that organizations experience knowledge depreciation. "Knowledge acquired through learning by doing is found to depreciate rapidly" and "failure to allow for depreciation of learning may result in forecasts with large errors" (Darr et al. 1995, pp. 17501751). When a knowledge worker leaves a firm, the organization's knowledge base changes, with some depreciation inevitable either through decay or obsolescence.

Knowledge decay with respect to human capital has been established from both cognitive and strategic management perspectives. Cognitive knowledge is dependent on human memory, and memory decays over time in numerous ways (Schacter 2001). Knowledge decay can occur from transience, absent-mindedness, blocking, misattribution, suggestibility, bias, or persistence. Organizations can also forget or lose track of existing knowledge (Argote et al. 1990; Darr et al. 1995). Computer systems are considered to experience knowledge decay from the attrition of operational workers who are tasked with the maintenance of complex computer systems (Fielden 1990).

Knowledge may also lose value gradually as it becomes commonplace or obsolete. As a source of sustaining competitive advantage, knowledge begins to lose value once it is codified (Nonaka and Takeuchi 1995); that is, it begins to be shared, transferred, sold, or stolen-diluting its novelty and scarcity and, hence, its competitive value. For example, once the competition copies unique capabilities, the market value generally is significantly diluted. Obsolescence of skills and knowledge, turnover, and retirement can all act to deplete the human resource of the organization (Flamholtz and Lacey 1981). We believe that obsolescence poses the greater risk to sustaining competitive advantage as it is dependent upon the complexity and externality of the market. Within computational knowledge bases (Debenham 2000), knowledge obsolescence degrades knowledge integrity by failing to update a knowledge base as new, requisite knowledge appears.

For our model, a worker's competence will deteriorate from period to period with the depreciation rate $(\delta)$ where $\delta \in[0,1]$. At the same time, a worker's competence will be enhanced by participating in $\mathrm{KC}$ processes (from $\mathrm{G}_{i j t}$ ), which acts as a basic incentive for a worker to have an individual gain on competence (human capital). (Other incentives, e.g., compensation for participation, promotion, etc., can be considered in a more sophisticated formulation.) While competence cannot exceed 100 percent (i.e., $\varepsilon_{i t}=1$ specified later in constraint (17)), a demonstrated competence of the worker $i$ for performing task $j$ at the beginning of time period $t$ can be formulated as (with a noise term $\phi$ ):

$$
\varepsilon_{i j t}=(1-\delta) \times \varepsilon_{i j(t-1)}+G_{i j(t-1)}+\phi
$$

\section{Employee Contracts}

The employee contract is motivated by the need to establish a hierarchical governance structure 
which differentiates the organization and creates competitive advantage. Hierarchical governance is an assumption of transaction cost economics (Coase 1937) where specialized tasks are associated with transactions and, by the nature of the employment contract, associated with workers to optimize the collective skill set to accomplish the defined tasks (Williamson 1980). Without the need for organizational differentiation, a market orientation would be preferred and the employee contract would be replaced by a supply chain orientation, pure market, or auction structure. Differentiation of the organization infers that a group orientation for achieving organizational knowledge creation is preferred. Otherwise, individual workers gaining new knowledge optimize their own task responsibilities, and personal goals are not necessarily consistent with the goal of the organization.

When the risk levels for desired benefits or results are high or uncertain (as is often the case for knowledge creation strategy), the employment contract is superior to the market (or sales) contract (Simon 1951). This conclusion shows consistency with causal ambiguity as a requirement for sustained competitive advantage (Barney 1991). Therefore, worker salaries can be considered as an expense toward providing causally ambiguous resource advantages for the firm, leading to sustained market advantage. Knowledge creation processes that are inherently strategic seek innovation and, in such cases, the desired benefits or results are not known with certainty.

Agency theory (Jensen and Meckling 1976) considers discrepancies in motivation between agents (e.g., knowledge workers) and the firm (i.e., owners) with regard to goals and objectives. Aligning worker task performance to those most conducive to the objectives of the firm (i.e., firm profit maximization) requires incentives, which are designed to consider the individual interests of the worker. Agency theory assists in explaining why informal knowledge creation processes, such as informal communities of practice, may be less efficient for the firm than would formal KC processes, as individuals will choose those processes that maximize their own self-interest. It also suggests that aligning worker motivation to firm objectives with regard to KC processes may require additional incentives, particularly with respect to group performance and long-term organizational goals.

We classify a worker's compensation received from an organization into two different types: skilloriented (fixed compensation) and task-oriented (variable compensation). Fixed compensation $\left(C^{f i x e d}\right)$ for a worker is based on a worker's demonstrated competence levels for tasks with a corresponding market value at the beginning of a time period $t$, and variable compensation ( $C^{\text {variable }}$ ) for a worker is based on the contribution of the worker toward performing task(s) during the time period $t$. Therefore, in time period $t$, the organization's compensation for workers can be defined as:

$$
\begin{gathered}
C^{f i x e d}=\sum_{i=1}^{I} \sum_{j=1}^{J} M V_{j} \times \varepsilon_{i j t} \\
C^{\text {variable }}=\sum_{i=1}^{I} \sum_{j=1}^{J} C_{i j t}
\end{gathered}
$$

For our model, the value of performing task $j$ by worker $i$ during time period $t$ is determined by the base value for completing a task $\left(C^{t}\right)$ and the portion of time a worker $i$ devotes to the task $\left(X_{i j t}\right)$ :

$$
C_{i j t}=C^{t} \times X_{i j t}
$$

The $M V_{j}$ represents the unit market value of a worker's competence for performing task $j$. We assume that $M V_{i}$ is fixed in the short run and can be generally applied to each worker. $C^{\text {variable }}$ is the total compensation for the portion of time that workers in an organization devote to performing task(s).

\section{Organizational Synergy and Complementarity}

Synergy allows for a greater increase in benefit from differentiation, or in a decrease in costs, than would be expected from the mere summation of 
individual efforts. Organizational synergy may be viewed in terms of substitutable resources or coordinated resources. Substitutable resources in a study of the manufacturing industry (St. John and Harrison 1999) showed no significant contribution to superior cost savings or improved competitive position; resource coordination did. In Barua et al. (1996), resource coordination, attributed to complementarity, was shown to be necessary for successful reengineering projects.

Complementarity theory (Edgeworth 1881) describes the economic value of leveraging a resource into different domain applications. Teece (1984) extends complementarity to consider how a resource can have an effect on another resource. Milgrom and Roberts (1995) demonstrate that complementarity is supermodular (i.e., the benefits to the group exceed the sum of individual resources). In applying complementarity to human resources, Lauren and Foss (2003) discuss two considerations relating to human resources complementarity: (1) that it leads to a reduction of effort by eliminating redundant tasks, and (2) that complementarity is a source of path dependence. Knowledge workers address complex task sets, i.e., a set decomposing into various discrete tasks which we could label $A, B, \ldots . n$. In the first instance, human resources complementarity can occur when person A performs knowledge task $A$ which benefits one or more other human resource, such as sharing a literature scan among a group of researchers. The ability of one worker to forego a task that has already been accomplished by a coworker for another task set allows the organization to acquire the revenue for both task sets without penalty.

The second consideration acknowledges that this interaction among resource groups introduces path dependence among the resources. Wade and Hulland (2004, p. 123) use complementarity theory relating that firm resources "rarely act alone in creating or sustaining competitive advantage," and Harrison et al. (2001) showed positive strategic management performance by applying complementarity in the elimination of redundant tasks. Thus, a manager needs to consider the availability of worker competence as well as the timing of KC processes in order for individual efforts to maximally benefit group outcomes and organizational competitive advantage.

Complementarity squarely focuses the unit of analysis at the group level. Complementarity provides more specificity guiding how human resource development should occur; it directs the organization to optimize group performance beyond the sum of individual performance. Combining RBV and complementarity, the organization considers the human resource as adaptable rather than dispensable. The human resource can, individually, improve upon its current state. Complementarity increases this value supermodularly (i.e., beyond the sum of the individual workers).

In our model, we define revenues $(R)$ of an organization as the performance on tasks based on workers' contributions. Increasing worker competence helps an organization to compete in the business environment in various ways. On the individual level, the benefit is measured by the efficiency and output of completing those tasks acceptably. We consider the required competence to complete a task $j\left(L_{j}\right)$ and the actual competence possessed by worker(s) for the task. If an organization's workers perform tasks but the workers' competence cannot satisfy the required competence for the task, it is intuitive to assume that these tasks will not be performed as desired. Therefore, the true benefits from the performed tasks should account for the discrepancy of the workers' competence for failure and error. On the other hand, if an organization's workers perform a task and the workers' competence exceeds the required competence for the task, the organization will benefit from better efficiency and productivity. On the organization level, supported by the theory of complementarity, we use a markup level to represent the additional benefit generated by organization-wide competence as a competitive advantage. Therefore, an organization's benefit revenue should be formulated as

$$
R=\left(1+M_{t}^{T}\right) \times\left(\sum_{j=1}^{J}\left(\left(\sum_{i=1}^{I} C_{i j t}\right) \times\left(\frac{\sum_{i=1}^{I} \varepsilon_{i j t} \times X_{i j t}}{L_{j}}\right)\right)\right)
$$


$M_{t}^{T}$ is the indicator for the benefit resulting from the organization-wide competence level. For instance, IBM can charge a premium for its services because of its well established reputation (supported by its highly trained workforce) that would not be generally possible for a small company. It can be described as a function $(g)$ such as $M_{t}^{T}=g\left(\sum_{i=1}^{t} \sum_{j=1}^{J} \varepsilon_{i j t}\right)$. We conjecture that the marginal increase of the indicator is relatively small and increases slowly when the organization-wide competence level is small. The marginal increase of the indicator is relatively large and increases quickly when the organization-wide competence level is large. Also, the indicator increases when the organization-wide competence level increases. Therefore, $g(\cdot)$ is in the form of a strictly increasing exponential function.

$$
g\left(\sum_{i=1}^{I} \sum_{j=1}^{J} \mathcal{E}_{i j t}\right)=\lambda \times e^{\theta \times \sum_{i=1}^{\prime} \sum_{j=1}^{J} \varepsilon_{j r}}
$$

Constants, $\lambda>0$ and $\theta>0$, are assigned, and $e$ is the base of the natural logarithm. Managers can alter the value of constants $(\lambda, \theta)$ for a specific functional form for $g(\cdot)$ in order to investigate how organizational-wide competence levels affect an organization's strategy. For example, adjusting constant $\lambda$ affects the magnitude of the indicator and adjusting constant $\theta$ affects the slope of marginal increase of the indicator. Therefore, if a manager believes that the magnitude of impact of the organization-wide competence to organizational benefit is relatively small, the manager can set constant $\lambda$ lower. If a manager believes that the impact of the organizational benefit from a larger organization-wide competence should be much larger than that from small organization-wide competence, the manager can set constant $\theta$ higher.

\section{Specific Knowledge Creation Process Costs}

Specific KC process costs are those typically considered for $\mathrm{KC}$ processes, which include direct costs, such as training fees or tuition, travel and living expenses, and any required materials that must be purchased to participate in the $\mathrm{KC}$ process. These costs are direct and measurable.

Human capital theory (HCT) addresses conditions under which employer and worker share various training cost allocations depending on a variety of factors (Becker 1962). HCT has been demonstrated to empirically relate to information technology professionals (Josefek and Kauffman 1999). Training can be considered general (the benefits apply equally to a market set of employers) or specific (the benefits contribute predominantly to a specific organization). The inducement for the worker to cover any of the cost of general training is tied to the security provisions in the employment contract and dilution of these provisions reduces incentives for employees to bear these costs. Workers will reject requirements that they pay for general training costs if employment contracts are not specific (Malcomson et al. 2003). For example, Java programming language skills have a general market value (and under $\mathrm{HCT}$, workers bear the training costs), but a worker will resist paying for Java training if a Microsoft career is preferred and current employment is not ensured. So, while general training provides a general benefit to the worker, employers are obligated, at least, to provide a benefit on the training (e.g., increased wages) in order to retain workers, or to ensure that $\mathrm{KC}$ processes maximally benefit the organization.

Under $\mathrm{HCT}$, a firm should terminate a loyal worker when future productivity is uncertain and hiring costs are low; however, under HCT, market conditions (and affected tasks) are static, not dynamic, such as has occurred in the recent e-commerce/ e-business market. HCT also ignores the effects of complementarity on organizational productivity where workers affect, and are affected by, the organizational context and group dynamics. Therefore, careful assessment must be made by the firm's management with regard to expectations and policies regarding worker retention and development.

In our model, the cost of participating in $\mathrm{KC}$ processes $\left(C^{\text {training }}\right)$ is considered. We assume that 
the cost of a KC process is linear to the intensity of the $\mathrm{KC}$ process $\left(v_{j}\right)$. Workers may participate in a $\mathrm{KC}$ process at a fractional level, denoted by $Y_{i j}$. Therefore, with the base cost of a $\mathrm{KC}$ process, denoted as $C^{K C}$, the cost of participating in $K C$ processes can be represented as:

$$
C^{\text {training }}=\sum_{i=1}^{l} \sum_{j=1}^{J} C^{K C} \times v_{j} \times Y_{i j t}
$$

\section{Opportunity Costs}

Opportunity costs must be considered for organizational $\mathrm{KC}$ valuation as the investment in a specific KC process precludes that investment from being applied to another KC process or task. Transaction cost economics (Coase 1937) relates to asset specificity in this regard and the degree to which the asset (worker) can be redeployed for other purposes without a loss of value; opportunity cost becomes the reduction in value that will occur from such redeployment. Decision makers with greater knowledge may discount risk associated with near-term opportunity costs more often than will those with less knowledge (Vera-Munoz 1998), and opportunity costs may be valued more when posed in a personal context than in a business context.

A trade-off between short-term, rent-seeking opportunity and entrepreneurial opportunity has also been noted, where the organization's need to maximize short-term benefits must be balanced with the long-term investments necessary to obtain future market leadership (Baland and Francois 2000). Interest rates and recruitment costs have been considered as opportunity costs for not training (Stevens 1994). Failure and errors have been highlighted as the largest opportunity costs for not training (Hubbard 1995).

Taken in the context of organizational $\mathrm{KC}$ and human capital theory, workers would be expected to use personal time (and possibly expense) to obtain general training. Certain leisure activities, though, may have utility beyond a person's wage (Shaw 1992). If we consider that many knowledge worker tasks are intense and draining, if not overly stressful, workers may easily choose a seasonal (time-sensitive) leisure activity such as fishing or spending time with their children, over additional regular wages for additional work. Therefore, traditional assumptions of individual utility that equate the cost of leisure time to no more than an individual's established wage rate would not hold.

A negative relationship for termination costs has been discovered in relation to a firm's willingness to provide on-the-job training. Booth et al. (2002) identified that employees were less willing to terminate their employment with those firms providing training than firms requiring employees to obtain training using their own resources. Thus, opportunity costs should be assessed along multiple dimensions. First, immediate opportunity costs should frame the basic input into our model. However, there are enterprise and longitudinal aspects to consider when assessing opportunity costs. If the enterprise, in general, supports a level of KC not supported within a specific department (organization), that organization cannot ignore the consequences of reducing what may be perceived as an expected investment. Second, the history of organizational decisions must be considered when calculating opportunity costs. When organizations have established $\mathrm{KC}$ process norms, workers may be expected to resent reductions in these investments; however, workers are more likely to forego KC process opportunities in the short-term when presented with a compelling justification.

Conservatively, in our model the opportunity cost $\left(C^{\text {opportunity }}\right)$ for an organization in a time period is the potential lost benefit from the portion of time in the specific time period where workers participate in $\mathrm{KC}$ processes but could have contributed to performing tasks. Other opportunity costs, such as errors incurred by a worker not having the requisite competence for the assigned task, are taken into account in calculating the benefit, $\mathrm{R}$. $\mathrm{We}$, therefore, use the following equation to represent opportunity costs:

$$
C^{\text {opportunity }}=R \times \frac{\sum_{i=1}^{I} \sum_{j=1}^{J} Y_{j i t}}{\sum_{i=1}^{I} \sum_{j=1}^{J} X_{j i t}}
$$




\begin{tabular}{|c|c|}
\hline Notation & Description \\
\hline$v_{j}$ & intensity of the KC process for task $j$ \\
\hline$\varepsilon_{i j t}$ & demonstrated competence of the worker $i$ for task $j$ at the beginning of time period $t$ \\
\hline$G_{i j t}$ & increased competence for worker $i$ by taking $\mathrm{KC}$ process for task $j$ during time period $t$ \\
\hline$G_{0}$ & highest possible gain of knowledge from participating in any $\mathrm{KC}$ process \\
\hline$\delta$ & depreciation rate of a worker's competence from period to period \\
\hline$R$ & $\begin{array}{l}\text { revenues of an organization as the performance on tasks based on workers' } \\
\text { contributions }\end{array}$ \\
\hline$C^{t}$ & base value for completing a task \\
\hline$C_{i j t}$ & cost of performing task $j$ by worker $i$ during time period $t$ \\
\hline$L_{i}$ & level of competence needed to complete the task $j$ \\
\hline$M V_{i}$ & unit market value of worker's competence for task $j$ \\
\hline$X_{i j t}$ & fraction of worker is time devoted to performing task $j$ during time period $t$ \\
\hline$Y_{i j t}$ & fraction of worker is time devoted to KC process for task $j$ during time period $t$ \\
\hline$C^{K C}$ & base cost for a KC process \\
\hline$M_{t}^{T}$ & $\begin{array}{l}\text { indicator for benefit resulting from complementary organization-wide competence level } \\
\text { during time period } t\end{array}$ \\
\hline$\phi, \rho$ & noise terms \\
\hline
\end{tabular}

\section{Knowledge Creation Valuation Model}

Specific notations relating to our model are summarized in Table 2.

Formally developed from previous discussion, the benefit $\left(\pi_{t}\right)$ of an organization in a time period $t$ can be expressed as:

$$
\begin{aligned}
\pi_{t}= & R_{t}-C_{t}^{\text {fixed }}-C_{t}^{\text {var iable }} \\
& -C_{t}^{\text {training }}-C_{t}^{\text {opportunity }}
\end{aligned}
$$

Now, we can set up the optimization model for an organization (with the decision variables $X_{i j t}$ and $\left.Y_{i j t}\right)$ in a time span of $T$ as follows:

$$
\text { Maximize } \Pi=\sum_{t=1}^{T} \pi_{t}
$$

Subject to

$$
\sum_{j=1}^{J} X_{i j t}+\sum_{j=1}^{J} Y_{i j t}=1 \quad \forall i, \forall t
$$

$$
\begin{array}{ll}
X_{i j t}, Y_{i j t} \geq 0 & \forall i, \forall t \\
0 \leq G_{i j t} \leq 1-\varepsilon_{i j t} & \forall i, \forall t \\
0 \leq \varepsilon_{i j t} \leq 1 & \forall i, \forall t
\end{array}
$$

By inserting other formulas, equation (12) can be expressed as

$\pi=$

$$
\begin{aligned}
& \left(1+M_{t t}\right) \times\left(\sum_{j=1}^{J}\left(\left(\sum_{i=1}^{I} C^{t} \times X_{i j t}\right) \times\left(\frac{\sum_{i=1}^{L} \varepsilon_{i j t} \times X_{j t}}{L_{j}}\right)\right)-\right. \\
& \sum_{i=1}^{I} \sum_{j=1}^{J} M V_{j} \times \varepsilon_{i j t}-\sum_{i=1}^{I} \sum_{j=1}^{J} C^{t} \times X_{i t}-\sum_{i=1}^{I} \sum_{j=1}^{J} C^{K C} \times V_{j} \times Y_{i j t}- \\
& \left.\left(1+M_{t}^{T}\right) \times\left(\sum_{j=1}^{J} \sum_{i=1}^{I} C^{t} \times X_{i j t}\right) \times\left(\frac{\sum_{i=1}^{I} \varepsilon_{i j t} \times X_{i j t}}{L_{j}}\right)\right) \times \frac{\sum_{i=1}^{I} \sum_{j=1}^{J} Y_{i t}}{\sum_{i=1}^{T} \sum_{j=1}^{J} X_{i j t}}
\end{aligned}
$$

In order to consider more realistic future benefits of $\mathrm{KC}$ processes, we can calculate the net present value (NPV) of benefits for an organization in different time periods. Therefore, equation (13) can be modified as: 


$$
\text { Maximize } \Pi=\sum_{t=1}^{T} N P V\left(\pi_{t}\right)
$$

$N P V(x)=\frac{x}{(1+r)^{1}}$ where $r$ is the interest rate of the time period

\section{Hypotheses Development}

The simulation approach is often used in social science to address complex problems that cannot be solved by other means such as optimization modeling, survey, or case study. As defined by Bratley et al. (1987), simulation drives the model of a system with suitable inputs and observes the corresponding outputs. Further, simulation affords the opportunity to explore multiple situations ascertaining answers to research questions without negatively impacting the organization or individuals.

Our optimization model for assessing value in organizational knowledge creation is not directly solvable or tractable without human interaction. In our simulation, we manipulate external variables such as knowledge depreciation and time (which cannot be controlled in a natural setting) and observe how these variables affect organizational benefits while interacting with other variables that can be influenced by managers, such as skill to task alignment and $\mathrm{KC}$ process intensity. Our hypotheses development reflects behavior or expectations considered by many managers to be typical management practice.

\section{Skill to Task Match}

We consider three types of skill to task match: high, low, and random. A high match indicates a strong degree of alignment between the desired assigned task and the required worker skill level; a low match indicates a weak alignment. (A high match for all knowledge workers can produce an optimal contribution for an organization under specific circumstances, but there is little evidence to conclude that such optimization is typical in organizations.) A random match assumes a uniform distribution of workers' skills to assigned tasks ranging from high match to low match, and is indicative of the effects of indeterminate decision making. A high match implies that the KC process participation decision considers the organizational task requirements and complementarity benefits for the intended tasks. A weak match is indicative of a weak or naïve organizational KC process strategy. We can consider that a weak match (or, at best, a random match) exists when knowledge workers are expected to determine their own KC process participation and investment which may not result in organization benefits.

Should individual knowledge workers be the sole source of the KC process participation decision? Under human capital theory, individuals will maximize their personal marketability, but competitive advantage in an organization necessitates skills or skill groupings (with others) that do not exist in the general market. In practice, managers may leave the selection of KC process(es) to the discretion of the worker. Our hypothesis indicates this organizational expectation.

Hypothesis 1: Organizations do not maximize organizational returns when knowledge workers determine their own $\mathrm{KC}$ process(es).

\section{KC Process Intensity}

Can an organization reduce the number of $\mathrm{KC}$ processes in which a knowledge worker participates to improve upon the organization's revenue over time? The intensity with respect to $\mathrm{KC}$ process relates to the sophistication of the knowledge intended for the participant. The manager's KC process decision criteria goes beyond a simple binary alternative of deciding if a particular knowledge worker should participate in a specific KC process during some time period. One option relates to increasing the intensity of the KC process in an attempt to decrease the number of $\mathrm{KC}$ process participations. The rationale is that if there is more information provided, then the 
worker can participate in fewer KC processes and obtain the same net effect. If this is possible, then the organization reduces the delay or reduction of revenue that occurs when the worker is not productive with regard to the assigned tasks.

\section{Hypothesis 2: Increasing KC process intensity reduces the frequency of required $\mathrm{KC}$ process participation without risk to organization returns.}

\section{Knowledge Depreciation}

We equate low knowledge depreciation to a stable market or to positions similar to clerical or assembly type workers as traditionally defined. For these types of markets and positions, tasks are well defined, repetitive, and slow to change. We consider a market situation with respect to what has been called "the new economy" as an example of high depreciation. Managers and other knowledge workers need to adapt to constant change and strengthen their knowledge frequently.

How does the level of knowledge depreciation affect $\mathrm{KC}$ process investment decisions? We consider that high or medium knowledge depreciation characterizes the environment of the contemporary knowledge worker. Knowledge depreciation at a low level frames the industrial economy or operational workers' tasks. Thus, requirements for new knowledge may influence $\mathrm{KC}$ investment decisions depending upon the level of knowledge depreciation.

Hypothesis 3: Increases in knowledge depreciation will increase the need for $\mathrm{KC}$ process participation by knowledge workers.

\section{KC Process Selection}

In general, managers may consider KC processes, such as training, in one of three ways: as an entitlement (everyone deserves the same), as a bonus (those excelling receive it as a reward), or as a remedy (marginal workers receive it to bring them up to the group norm). The entitlement strategy affects KC process frequency and selection is commonly applied, but does it benefit the organization?

\section{Hypothesis 4: Organizations reduce organizational returns by applying an entitlement strategy with respect to $\mathrm{KC}$ process participation.}

\section{Longitudinal Effects of KC Processes}

Is it beneficial to invest in $\mathrm{KC}$ processes to the detriment of completing some short-term tasks? Typically, one may assume that $\mathrm{KC}$ process participation, because it always incurs a minimum of an opportunity cost, would negatively impact short-term organizational benefits, but ideally it bolsters longer-term benefits. The realities of aggressive competition often pressure managers into removing $\mathrm{KC}$ process opportunities to stay on task schedule. This reality becomes self-reinforcing when schedules are determined without ensuring adequate planning for $\mathrm{KC}$ process participation. In our simulation, we consider short(12 month), medium- (24 month), and long-term (60 month) time periods for assessing the impacts of the various $\mathrm{KC}$ process decisions and parameters.

Hypothesis 5: The pressure to meet short-term delivery requirements reduces long-term organizational value if $\mathrm{KC}$ process investments are eliminated.

\section{Analysis and Results}

In the previous sections, we developed a model reflecting the variables that affect the valuation of an organization in light of the ability to improve upon that value based upon the $\mathrm{KC}$ process participation of its knowledge workers. Following the development of this model, we hypothesized several conditions affecting decisions that a manager can make directly to impact organizational value and of external factors that must be 


\section{Table 3. Variables and Values Assigned During the Simulation}

\begin{tabular}{|l|l|l|}
\hline \multicolumn{1}{|c|}{ Variables } & Values Assigned & \multicolumn{1}{c|}{ Comments } \\
\hline $\begin{array}{l}\text { Knowledge } \\
\text { depreciation rate }(\delta)\end{array}$ & $\begin{array}{l}\text { Low }=0.01 \\
\text { Medium }=0.05 \\
\text { High }=0.10\end{array}$ & $\begin{array}{l}\text { For a depreciation rate of } 0.01 \text {, the competence of a } \\
\text { worker will decrease by } 1 \% \text { after one time period. }\end{array}$ \\
\hline $\begin{array}{l}\text { KC process } \\
\text { frequency }\end{array}$ & $\begin{array}{l}\text { No KC process } \\
\text { Every six months } \\
\text { Every one year }\end{array}$ & $\begin{array}{l}\text { No KC indicates workers do not participate in any KC } \\
\text { processes. Frequent (moderate) participation indi- } \\
\text { cates that each worker participates in a KC process } \\
\text { every other } 6 \text { (12) months. }\end{array}$ \\
\hline KC process cost $\left(C^{K C}\right)$ & $\begin{array}{l}\text { Low }=\$ 500 \\
\text { Medium }=\$ 1000 \\
\text { High }=\$ 2000\end{array}$ & $\begin{array}{l}\text { For a worker participating in a KC process with } \\
\text { intensity of "100\%" and base KC process cost of } \\
\text { "\$500" full time in a time period, the cost is } \$ 500 .\end{array}$ \\
\hline $\begin{array}{l}\text { Match of competence } \\
\text { with task requirement }\end{array}$ & $\begin{array}{l}\text { Low match } \\
\text { Random match } \\
\text { High match }\end{array}$ & $\begin{array}{l}\text { High match indicates that a worker will be assigned to } \\
\text { the task in which the worker's competence level is the } \\
\text { highest. }\end{array}$ \\
\hline $\begin{array}{l}\text { KC process intensity } \\
\left(v_{j}\right)\end{array}$ & $\begin{array}{l}\text { Low }=0.20 \\
\text { Medium }=0.50 \\
\text { High }=0.80\end{array}$ & $\begin{array}{l}\text { The intensity of a KC process for task } j \text { in any time } \\
\text { period is between } 0 \% \text { and } 100 \% \text { in the simulation. }\end{array}$ \\
\hline Base task cost $\left(C^{t}\right)$ & $\begin{array}{l}\text { Low }=\$ 2000 \\
\text { Medium }=\$ 4000 \\
\text { High }=\$ 8000\end{array}$ & $\begin{array}{l}\text { For a worker performing a task with required } \\
\text { competence of "100\%" and base task cost of "\$2,000" } \\
\text { full time in a time period, the cost is } \$ 2,000 .\end{array}$ \\
\hline Base skill cost $\left(M V_{j}\right)$ & $\begin{array}{l}\text { Low }=\$ 200 \\
\text { Medium }=\$ 400 \\
\text { High }=\$ 800\end{array}$ & $\begin{array}{l}\text { For a worker with competence of "1" and unit market } \\
\text { value of skill as "\$200" in a time period, the cost is } \\
\$ 200 .\end{array}$ \\
\hline
\end{tabular}

considered that also impact organizational value with regard to KC process participation. Specifically, with many possible variations of knowledge depreciation rate, task costs, skill costs, KC process frequency, $\mathrm{KC}$ process cost, task to skill match, and $\mathrm{KC}$ process intensity in a dynamic knowledge environment, we selected simulation as our research method to investigate the relationship and interaction of these conditions. Our approach recognizes the contextual nature of knowledge and allows for parameters to be modified as the organizational context changes.

\section{Simulation Setup}

We followed certain assumptions and set values for factors and parameters so that our simulation would reflect a real-world setting. These factors and the relevant values assigned to them for the simulation are summarized in Table 3 based on the following rationale. Monetary amounts (such as $\$ 500, \$ 1,000$, and $\$ 2,000$ ) are chosen as reasonable real-world examples, especially with respect to the high-technology industry. Specific organizations may have different parameters; however, we are interested in observing trends or patterns influencing organizational value under each scenario. This approach is structured to assist theory-driven research. In addition, we perform various sensitivity analyses on parameter impacts.

We developed and implemented a simulation program using Visual Basic. Pseudo code of the simulation runs is provided in Table 4 . We simulated 100 runs for each of the 2,187 combinations to ensure comparability across different scenarios of the selected variables: initial worker competence on different tasks, required competence for different tasks, and time periods that workers are assigned to participate in $\mathrm{KC}$ pro- 


\section{Table 4. Simulation Pseudo Code}

\section{Simulation Runs}

Section $\mathbf{C}$ is looped for 12,24 , or 60 times for simulation of 1,2 , and 5 years

Section B through Section D are looped for 100 times and mean values of NPV are calculated

Section A through Section D are looped for 729 times for different combination of factors

(high/medium/low depreciation rate, high/random/low skill-task match, high/medium/low task cost, high/medium/low skill cost, high/medium/low KC process cost, high/medium/low KC process intensity)

$\mid \begin{aligned} & \text { Section A } \\ & \text { Set values for parameters and variables based on different scenarios } \\ & \text { Section B } \\ & \text { Set random number seed } \\ & \text { Initialize settings of initial workers' competences on different tasks, required } \\ & \text { competence for different tasks, and time periods that workers are assigned to } \\ & \text { participate KC processes for the beginning of first time period } \\ & \text { Section C } \\ & \text { Assign proportion of time that workers devote for tasks and KC processes for } \\ & \text { current time period } \\ & \text { Assign number of tasks and number of KC processes (if any) that a worker will take } \\ & \text { for current time period } \\ & \text { Assign the task(s) and the KC process(es) (if any) that a worker will take for current } \\ & \text { time period } \\ & \text { Calculate task-based compensation for workers } \\ & \text { Calculate skill-based compensation for workers } \\ & \text { Calculate cost for workers' participation of KC processes (if any) } \\ & \text { Calculate revenue generated from task performance } \\ & \text { Calculate opportunity costs of workers' time for not performing tasks } \\ & \text { Calculate profit generated from current time period } \\ & \text { Derive workers' competences on different tasks at the end of current time period } \\ & \text { (for the beginning of next time period) with consideration of knowledge } \\ & \text { depreciation and gain from KC processes (if any) }\end{aligned}$

cess(es). For each scenario, we used the mean of the net present values (NPV) from 100 runs to avoid possible random effects. We also used these mean values to perform statistical tests.

In the simulation, we reflected one time period as one month and we ran 12,24 , and 60 time periods (representing 1, 2, and 5 years) for different scenarios. We set 10 workers, 12 tasks, and 12 $\mathrm{KC}$ processes for the simulation. A worker's initial demonstrated competence on a specific task was assigned with 20 percent probability to be 0.8 , with
60 percent probability to be 0.5 , and with 20 percent probability to be 0.3 respectively (plus a noise value $\phi$ ). Tasks have a required competence level, $\left(L_{j}\right)$, set at $1.5,1.0$, and 0.5 to reflect task difficulty, allocated proportionately as 20 percent, 60 percent, and 20 percent, respectively. Market values $\left(M V_{j}\right)$ of competences for tasks were set to be linearly correlated with the required competence level $\left(L_{j}\right)$.

In the simulation, we operationalize the "high match" scenario as worker assignment for an 
available task where the worker's competence level to a specific task is the highest. A "low match" scenario indicates that a worker will be assigned to the task in which the worker's competence level of that specific task is the lowest. The "random match" scenario denotes that a worker will be randomly assigned to certain task regardless of the worker's competence level for that task. Each worker can perform up to three tasks and participate in up to three $\mathrm{KC}$ processes in a time period. ${ }^{6}$ A worker's first time period for participating in a $\mathrm{KC}$ process is randomly decided but each worker will participate in a $\mathrm{KC}$ process every other 6 or 12 time periods. In addition, when a worker's KC process time period occurs, this worker has to allocate at least 50 percent of the available time for the $\mathrm{KC}$ process in that time period. The base task cost $\left(C^{t}\right)$ and base skill cost $\left(M V_{j}\right)$ are set up so that the annual compensation for a worker will be approximately $\$ 40,000$, $\$ 80,000$, and $\$ 160,000$ for $\operatorname{low}\left(C^{t}\right)-\operatorname{low}\left(M V_{j}\right)$, medium $\left(C^{t}\right)$-medium $\left(M V_{j}\right)$, and high $\left(C^{t}\right)$-high $\left(M V_{j}\right)$ scenarios respectively. For each run in the simulation, we calculated the net present value with the interest rate of 0.005 for a time period (to reflect a 6 percent annual interest rate).

To obtain a corresponding range of values for the indicators $\left(M_{t}^{T}\right)$ as $(0,6)$ with an exponential resulting trend based on organization-wide competence, we set $\lambda$ as 0.5 and $\theta$ as 0.04 in our simulation. We ran additional simulations for sensitivity analysis of the effects on organizational benefits from $M_{t}^{T}$. We found that the observed results from these additional simulations identically and statistically match the resulting trends presented in the manuscript. That is, obtaining a corresponding range of values for the indicator $\left(M_{t}^{T}\right)$ as $(0,3),(0,6)$, or $(0,10)$ and setting

\footnotetext{
${ }^{6}$ Theory-based research has not yet generalized optimal task or KC process limits for knowledge workers. Therefore, we conducted sensitivity analysis by restricting a worker to (1) up to two tasks and up to two $\mathrm{KC}$ processes and (2) up to four tasks and up to four KC processes in one time period. Resulting trends (similar to those presented in Table 5 and Table 6) from these two types of restriction remain the same as those with the setting described above (up to three tasks and three $\mathrm{KC}$ processes).
}

parameters $\lambda$ and $\theta$ as $(\lambda=0.5, \theta=0.03),(\lambda=0.5$, $\theta=0.04)$, or $(\lambda=0.5, \theta=0.05)$, respectively, derived the same major resulting trends for measuring organizational benefits.

For the $f(\cdot)$ function of the learning effect from devoted time, we set parameters $\alpha$ as 100 and $\beta$ as 10 to get a mapping of a $(0,1)$ range (percentage of time devoted to $\mathrm{KC}$ process) to a $(0,1)$ range (learning effect) in a strictly increasing Scurve functional form. For the $O_{b}(\cdot)$ function of the learning effect from a basic KC process, we set parameters $\alpha^{\prime}$ as 0.01 and $\beta^{\prime}$ as -10 in order to get a $(0,1)$ range (i.e., existing worker competence on the task) to a $(1,0)$ range (i.e., learning effect) mapping in a strictly decreasing S-curve functional form. Finally, for the $O_{a}(\cdot)$ function of the learning effect from an advanced KC process, we set parameters $\alpha "$ as 100 and $\beta "$ as 10 in order to get a $(0,1)$ range (i.e., existing-worker-competenceon-the-task) to a $(0,1)$ range (i.e., learning-effect) mapping in a strictly increasing S-curve functional form.

\section{Simulation Results}

We simulated 100 runs for each of the 2,187 combinations of variables on two primary scenarios to investigate a manager's decision-making alternatives with respect to KC process participation. Our results consider the derived organizational benefits in the form of net present value (NPV) with the use of equation (19). In Scenario I, we examined the effects (with respect to organizational value) of the depreciation rate and the skill on task alignment (i.e., match) over time controlling for $\mathrm{KC}$ process costs and $\mathrm{KC}$ intensity. In Scenario II, we examined the effects of the depreciation rate on $\mathrm{KC}$ intensity alignment with respect to organizational value over time, controlling for $\mathrm{KC}$ process costs, task costs, and skill costs.

\section{Scenario I}

Table 5 summarizes the simulation results for Scenario I. In this scenario, the impact on KC pro- 


\section{Table 5. Scenario I: Effects of Skill to Task Alignment, Knowledge Depreciation, and} Time

\begin{tabular}{|c|c|c|c|}
\hline & Control Variables: & KC Costs = Medium & KC Intensity = Medium \\
\hline Depreciation = High & 12 time periods & 24 time periods & 60 time periods \\
\hline $\begin{array}{l}\text { Match High } \\
\text { Match Random } \\
\text { Match Low }\end{array}$ & $\begin{array}{l}e 6 p>e 12 p>N K C \\
N K C>e 12 p>\text { e6p } \\
\text { NKC }>\text { e12p }>\text { e6p }\end{array}$ & $\begin{array}{l}e 6 p>\text { e12p }>\text { NKC } \\
\text { e12p \& e6p }>\text { NKC } \\
\text { NKC }>\text { e12p }>\text { e6p }\end{array}$ & $\begin{array}{l}e 6 p \& \text { e12p }>\text { NKC } \\
\text { e12p }>\text { e6p }>\text { NKC } \\
\text { NKC }>\text { e12p }>\text { e6p }\end{array}$ \\
\hline Depreciation $=$ Medium & 12 time periods & 24 time periods & 60 time periods \\
\hline $\begin{array}{l}\text { Match High } \\
\text { Match Random } \\
\text { Match Low }\end{array}$ & $\begin{array}{l}e 6 p>\text { e12p }>\text { NKC } \\
\text { NKC }>\text { e12p }>\text { e6p } \\
\text { NKC }>\text { e12p }>\text { e6p }\end{array}$ & $\begin{array}{l}\text { e6p }>\text { e12p }>\text { NKC } \\
\text { e12p }>\text { e6p }>\text { NKC } \\
\text { e12p }>\text { e6p }>\text { NKC }\end{array}$ & $\begin{array}{l}e 12 p>\text { e6p }>\text { NKC } \\
\text { e12p }>\text { e6p }>\text { NKC } \\
N K C>\text { e12p }>\text { e6p }\end{array}$ \\
\hline Depreciation = Low & 12 time periods & 24 time periods & 60 time periods \\
\hline $\begin{array}{l}\text { Match High } \\
\text { Match Random } \\
\text { Match Low }\end{array}$ & $\begin{array}{l}e 6 p>e 12 p>N K C \\
N K C>e 12 p>e 6 p \\
N K C>e 12 p>e 6 p\end{array}$ & $\begin{array}{l}e 6 p>\text { e12p }>\text { NKC } \\
\text { e12p > e6p \& NKC } \\
\text { e12p > e6p > NKC }\end{array}$ & $\begin{array}{l}e 12 p>\text { e6p }>\text { NKC } \\
\text { e12p }>\text { e6p }>\text { NKC } \\
\text { e12p }<\text { e6p }>\text { NKC }\end{array}$ \\
\hline
\end{tabular}

$\mathbf{e} 6 \mathbf{p}=\mathrm{KC}$ process participation after every 6 time periods; $\mathbf{e 1 2 p}=\mathrm{KC}$ process participation after every 12 time periods; NKC $=$ No KC process participation. Note: All comparisons with ">" indicate that paired two sample $t$-tests for means yielding $p$ values $\leq 0.1$.

cess participation is influenced by skill to task match (high, random, or low), assessment interval $(12,24$, and 60 time periods which we equate to months), and knowledge depreciation rate (high, medium, or low). Control variables are KC process costs and $\mathrm{KC}$ process intensity (both set to medium). Note that we equate certain groups (using "\&") where paired two sample $t$-tests for means yield statistically insignificant results (i.e., $p$ $\geq 0.1$ ). In other words, these are the situations where the two combined groups show no significant difference from each other with respect to the organizational benefits they provide.

First, we examine our model to identify observations that are reasonably consistent and relevant. For instance, we find that organizational benefits are better when task values (which become a revenue source to the organization) are high and skill costs are low (other than a few exceptions discussed below). This observation holds true independently of how often a worker participates in a KC process (every 6 or 12 months, or not at all), and it holds true longitudinally (over 12,24 , or 60 months). Additionally, we find that organizational value is worse when task values are low but when the applied skills require a high cost. Some interesting exceptions occur when (1) knowledge depreciation is high and there is no $\mathrm{KC}$ process over the long term (60 periods) or (2) skill to task match is low over intermediate and long-term (24 and 60 ) periods. For these exceptions, organization benefits are better when both task values and skill costs are low and are worse when both task values and skill costs are high. When depreciation is high and there is no $\mathrm{KC}$ process over the long term, workers' competence deteriorates dramatically and too many knowledge workers must dedicate their efforts to a high value task. Under this circumstance, even when workers are assigned to tasks with a high match, the efficiency and output of performing these tasks will be very low (i.e., the revenue source is low). When the skill to task match is low over the medium or long term, workers assigned to tasks actually do not produce enough efficiency and output in performing tasks to cover the high variable cost $C^{\text {variable }}$. Therefore, for these 
exceptions, limiting the task value to lower the variable cost $C^{\text {variable }}$ (with low skill compensation) actually becomes desirable.

For Scenario I (refer to Table 5), when we manipulate knowledge depreciation on the skill to task match (alignment), under conditions of high knowledge depreciation, frequent $\mathrm{KC}$ process participation improves organizational returns in all time frames (short, intermediate, and long term) whenever a high skill to task match can be achieved. In this case, the productivity from the $\mathrm{KC}$ process offsets any revenue reduction due to the productivity delay which occurs when the workers are participating in the KC process(es). These results change for other conditions. Under high knowledge depreciation, but when the match between skill and task is random or low, no KC process participation is the preferred option in the short and intermediate term (i.e., 12 and 24 time periods). In these situations, workers are unable to compensate for the high rate of knowledge depreciation and the organization will not benefit with a low or random skill to task match. However, infrequent $\mathrm{KC}$ process participation (i.e., e12p) does provide a benefit in the longer term, suggesting that workers may eventually absorb the benefits of some KC process participation for longer-term organizational returns. Frequent KC process participation (i.e., e6p) results in an overinvestment under these parameters.

Under conditions of medium knowledge depreciation, frequent $\mathrm{KC}$ process participation continues to provide better organizational returns in all time frames (short, intermediate, and long term) whenever a high skill to task match can be achieved. For random match conditions, no KC process participation is preferred for the short term. Over time, infrequent KC process participation becomes the most beneficial. Under low match conditions, no $\mathrm{KC}$ process participation becomes the optimal decision for all time frames.

Consistent with the high and low knowledge depreciation alternatives, under conditions of low knowledge depreciation, frequent $\mathrm{KC}$ process participation once again becomes the best decision over all time frames (short, intermediate, and long term) to provide better organizational returns when the goal is a high skill to task match. For random or low skill to task alignment, no KC process participation provides better short-term organizational returns, but infrequent $\mathrm{KC}$ process participation benefits the organization in the intermediate to long term.

We can examine the longitudinal results (in net present value) by referring to Figure 2 , which depicts the organizational return over the duration of our three time frames (after 12,24, and 60 time periods). The figure shows the differences to organizational returns depending upon the impact of knowledge depreciation (high, intermediate, or low) and with respect to a medium skill to task cost. The charts on the left consider infrequent KC process participation (every 12 periods); those on the right consider frequent $\mathrm{KC}$ process participation (every 6 periods). Actual value levels (given the input parameters from Table 3) are noted over time.

Under all conditions of knowledge depreciation, organizational returns are better for high skill to task alignment when $\mathrm{KC}$ process participation occurs, with a relatively consistent positive slope. Under high knowledge depreciation, both random and weak matches exhibit negative slope (i.e., declining organizational value) over time. As noted earlier in Table 5, neither KC process option (frequent or infrequent) is desirable; more value accrues to the organization when it has no $\mathrm{KC}$ processes under high knowledge depreciation if the organization cannot achieve a high skill to task match. Under conditions of medium knowledge depreciation, the slope for a random match improves, but is relatively flat, while the slope for a weak match continues to decline. Under conditions of low knowledge depreciation, all slopes are positive, although those for a random or weak match are much less steep than are those for a high match, and they begin to flatten over the long term. Under these conditions, as long as skill to task costs are moderate (or nominal), the organization will be profitable, but not necessarily competitive (against competing organizations able to attain a high skill to task match without increasing their cost). 


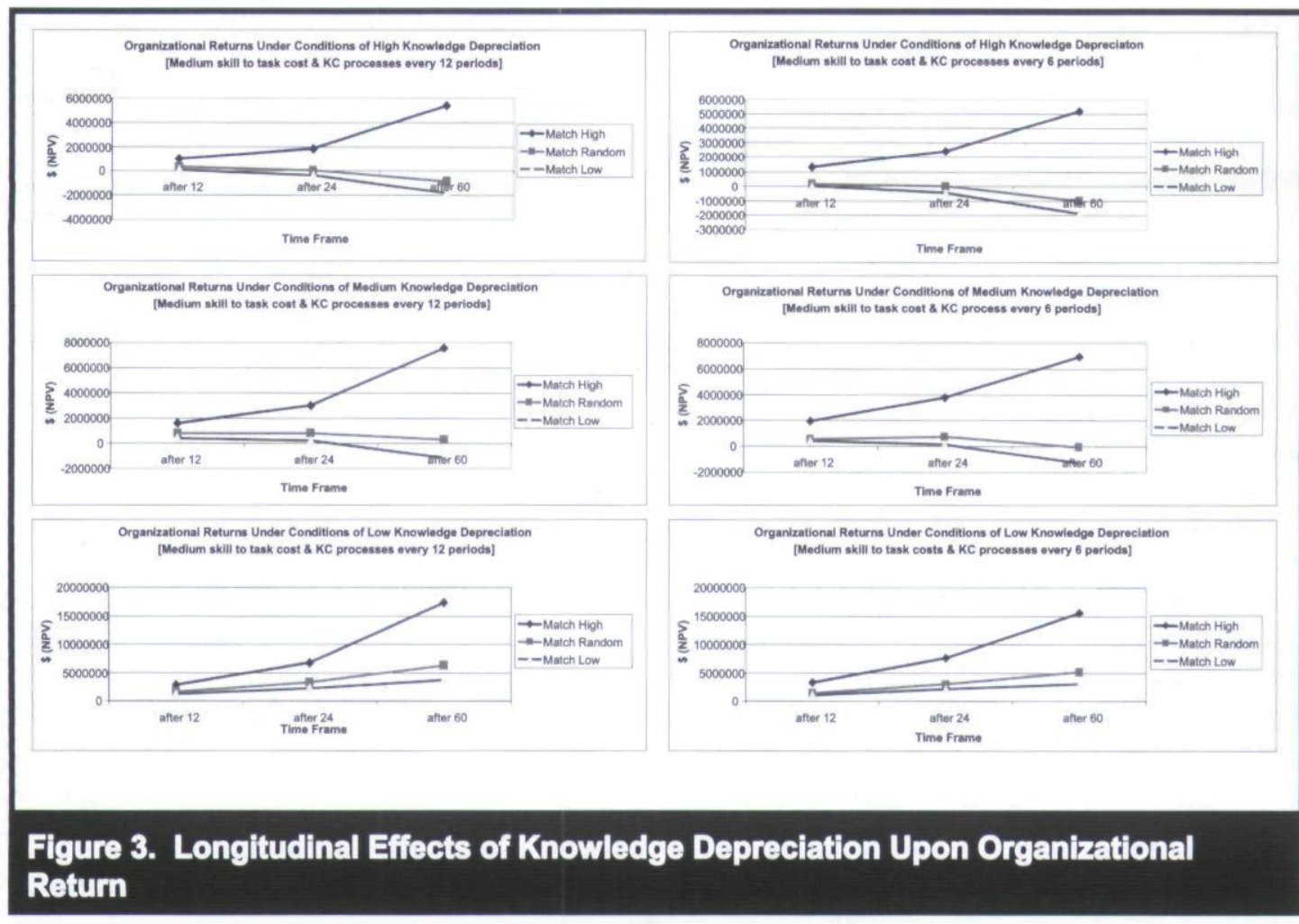

In assessing the entire set of charts, the level of knowledge depreciation impacts the actual value available to the organization; the higher the depreciation, the lower the organizational value regardless of whether the organization implements the best KC process strategy or not. With regard to the frequency of $\mathrm{KC}$ process participation, frequent $\mathrm{KC}$ participation results in higher value which increases over time and for most high match situations is independent of the level of knowledge depreciation.

\section{Scenario II}

Table 6 summarizes the simulation results for Scenario II. Under different knowledge depreciation rates and time frames, we are examining the ability to improve upon the organizational returns by manipulating $\mathrm{KC}$ process intensity.

Our results show little to no substantive change from Scenario I in KC process participation decisions. Under all conditions, the preferred KC process decision remains relatively constant, with the exception of low knowledge depreciation and high match in the long term. In that exception condition, any $\mathrm{KC}$ process participation is still preferable to no KC participation, but the increased KC process intensity appears to allow for infrequent $\mathrm{KC}$ process participation to become the preferred option. Under conditions of medium knowledge depreciation, high match, and a long-term time period, no significant difference was observed between frequent and infrequent $\mathrm{KC}$ process participation when $\mathrm{KC}$ process intensity is increased.

\section{Discussion}

In this section, we discuss the results in relation to our developed hypotheses, summarized in Table 7, which reflect the organization-level allocation decisions that need to be considered. Under simulation, we consider a wide range of alterna- 
Table 6. Scenario Il: Effects of Intensity, Skill to Task, Knowledge Depreciation, and Time

\begin{tabular}{|c|c|c|c|}
\hline \multirow{2}{*}{ Depreciation = High } & \multicolumn{3}{|c|}{ Control Variables: KC Cost, Task Cost, Skill Cost = All Medium } \\
\hline & 12 time periods & 24 time periods & 60 time periods \\
\hline Match High & $e 6 p>e 12 p>N K C$ & $e 6 p>e 12 p>N K C$ & $e 12 p>e 6 p>N K C$ \\
\hline Match Random & NKC $>$ e12p $>$ e6p & NKC $>$ e12p;> e6p & $e 12 p>e 6 p>N K C$ \\
\hline Match Low & NKC $>$ e12p > e6p & $N K C>$ e12p $>$ e6p & $N K C>e 12 p>e 6 p$ \\
\hline Depreciation = Medium & 12 time periods & 24 time periods & 60 time periods \\
\hline Match High & $e 6 p>e 12 p>N K C$ & $e 6 p>e 12 p>N K C$ & $e 12 p>e 6 p>N K C$ \\
\hline Match Random & NKC $>$ e12p > e6p & $e 12 p>N I C>e 6 p$ & e12p $>$ e6p $>$ NKC \\
\hline Match Low & NKC $>$ e12p $>$ e6p & $N K C>e 12 p>e 6 p$ & $N K C>$ e $12 p>$ e6p \\
\hline Depreciation = Low & 12 time periods & 24 time periods & 60 time periods \\
\hline Match High & $e 6 p>$ e12p > NKC & $e 6 p>e 12 p>N K C$ & e12p > e6p > NKC \\
\hline Match Random & $N K C>$ e12p $>$ e6p & $e 12 p>$ NKC \& e6p & $e 12 p>$ e6p > NKC \\
\hline Match Low & $N K C>$ e $12 p>$ e6p & e12p > e6p > NKC & $\mathrm{e} 12 p<\mathrm{e} 6 \mathrm{p}>\mathrm{NKC}$ \\
\hline
\end{tabular}

$\mathbf{e} 6 \mathrm{p}=\mathrm{KC}$ process participation after every 6 time periods; $\mathbf{e 1 2 p}=\mathrm{KC}$ process participation after every 12 time periods; NKC $=$ No KC process participation. Note: All comparisons with ">" indicate that paired two sample $t$-tests for means yielding $p$ values $\leq 0.1$.

tives to understand the many alternatives conceivable relating to $\mathrm{KC}$ process decision-making and its impact upon organizational returns. We find that the decision-making process is highly dependent upon aligning the skills of the knowledge workers to the organization's tasks, both with regard to competence alignment and with regard to organizational synergy. Competence allows for a reduction in workers per task due to increased efficiency and synergy allows for a reduction in the total number of discrete tasks due to complementarity that an organization must execute during a specified time frame.

\section{Skill to Task Match}

We observe that with a high match between skills and tasks (referring back to Tables 5 and 6 ) frequent KC process participation provides the best organizational profit. This observation holds over the short term (12 months) and longitudinally (24 and 60 months), and always holds true independently of the impact from knowledge depreciation (with the exception of a low match which suggests in the long term, 60 months, infrequent $\mathrm{KC}$ process participation is preferred over frequent $\mathrm{KC}$ process participation).

No KC process becomes a preference for random or low skill to task match in the short term independent of knowledge depreciation level, but as knowledge depreciation is reduced (to low or medium levels) or a longer-term evaluation period is invoked, infrequent $\mathrm{KC}$ process participation becomes a better decision to increase organizational benefit.

We find that these results help support hypothesis 1. In our model, organizational value is heavily affected by the interaction of knowledge depreciation and time. The opportunity to highly match knowledge workers' skills to assigned tasks is critical to maximize organizational value, and it allows for substantial differences in organizational value when such a high match can be sustained over time (see Figure 2). Additionally, in the absence of being able to achieve a high match, under conditions of high or medium knowledge depreciation, no KC process participation may be 


\section{Table 7. Hypotheses Summary}

\begin{tabular}{|c|c|c|c|}
\hline Hypothesis Description & Support? & Comments & $\begin{array}{l}\text { Construct } \\
\text { (Table 1) }\end{array}$ \\
\hline $\begin{array}{l}\text { 1: Organizations do not } \\
\text { maximize organizational } \\
\text { returns when knowledge } \\
\text { workers determine their } \\
\text { own KC process(es). }\end{array}$ & $\begin{array}{l}\text { Supported } \\
\text { refer to } \\
\text { Tables } 5 / 6 \\
\text { and Figure } \\
2\end{array}$ & $\begin{array}{l}\text { Individual knowledge workers can only } \\
\text { randomly match their skills (at best) with } \\
\text { organizational tasks unless they receive } \\
\text { organizational inputs (a KC process } \\
\text { itself). Agency theory supports that } \\
\text { isolated individual determination would } \\
\text { not result in high skill to task alignment. }\end{array}$ & $\begin{array}{c}\text { Competence and } \\
\text { Increased } \\
\text { Knowledge } \\
\text { (match level) }\end{array}$ \\
\hline $\begin{array}{l}2 \text { : Increasing } \mathrm{KC} \text { process } \\
\text { intensity reduces the fre- } \\
\text { quency of required } \mathrm{KC} \\
\text { process participation with- } \\
\text { out risk to organization } \\
\text { returns. }\end{array}$ & $\begin{array}{l}\text { Not } \\
\text { supported } \\
\text { refer to } \\
\text { Table } 6\end{array}$ & $\begin{array}{l}\text { Increasing KC process intensity shows } \\
\text { no substantial change in the decision } \\
\text { criteria of whether knowledge workers } \\
\text { participate in KC processes frequently, } \\
\text { infrequently, or not at all. }\end{array}$ & Intensity \\
\hline $\begin{array}{l}\text { 3: Increases in knowledge } \\
\text { depreciation will increase } \\
\text { the need for KC process } \\
\text { participation by knowledge } \\
\text { workers. }\end{array}$ & $\begin{array}{l}\text { Partially } \\
\text { supported } \\
\text { refer to } \\
\text { Figure } 2\end{array}$ & $\begin{array}{l}\text { Increases in knowledge depreciation } \\
\text { decrease overall organizational returns. } \\
\text { Under conditions of high knowledge } \\
\text { depreciation, the organization may not } \\
\text { benefit from KC process participation if } \\
\text { it can only achieve a random or low } \\
\text { match. }\end{array}$ & $\begin{array}{l}\text { Knowledge } \\
\text { Depreciation }\end{array}$ \\
\hline $\begin{array}{l}\text { 4: Organizations reduce } \\
\text { organizational returns by } \\
\text { applying an entitlement } \\
\text { strategy with respect to KC } \\
\text { process participation. }\end{array}$ & $\begin{array}{l}\text { Supported } \\
\text { refer to } \\
\text { Figure } 2\end{array}$ & $\begin{array}{l}\text { Organizational returns are highest only } \\
\text { when the skill to task match is high. } \\
\text { Sending everyone to all the same KC } \\
\text { processes is beneficial only when the } \\
\text { tasks are the same. }\end{array}$ & $\begin{array}{c}\text { Organizational } \\
\text { Synergy and } \\
\text { Complementarity } \\
\text { (selection) }\end{array}$ \\
\hline $\begin{array}{l}\text { 5: The pressure to meet } \\
\text { short-term delivery require- } \\
\text { ments reduces long-term } \\
\text { organizational value if } \mathrm{KC} \\
\text { process investments are } \\
\text { eliminated. }\end{array}$ & $\begin{array}{l}\text { Partially } \\
\text { supported } \\
\text { refer to } \\
\text { Tables } 5 / 6\end{array}$ & $\begin{array}{l}\text { Supported when knowledge worker skills } \\
\text { are well-aligned to organizational tasks } \\
\text { and for most conditions over time. Not } \\
\text { supported for short-term returns when } \\
\text { match is random or low. }\end{array}$ & $\begin{array}{l}\text { Longitudinal } \\
\text { Effects }\end{array}$ \\
\hline
\end{tabular}

the best option for maximizing organizational value. It is unlikely that employees would selfselect this option or, if they do, may not do so for the reasons assumed in our model. We allow for two primary worker participation alternatives in our model: task or KC process. Individual self interest can include a third: idle activity such as non-work related socialization or personal entertainment (e.g., personal web surfing).
So, by examining the results of our model and applying agency theory, we can see that a number of conditions will affect organizational value, for which individual self interest may contribute negatively or ignored. Therefore, it is highly unlikely that knowledge workers alone will choose the correct $\mathrm{KC}$ process strategy to maximize organizational value. Alternatively, we do not suggest that knowledge worker input should be ignored within 
the decision process. Our model (refer to Table 5 and Figure 2) suggests that there is a dramatic difference in organizational return between a high match and the other options. While we do not investigate the factors leading to a high match in this paper, it is unlikely that such a high match can occur without a significant interaction between the manager, who should represent the source of understanding of future organizational needs in addition to understanding the organizational value of demonstrated knowledge worker capabilities, and knowledge workers, who should also consider undemonstrated and potential capabilities into future task alignments.

Moving beyond our simulation, we can take a step back and consider the goals of managers with regard to their responsibilities to organizational performance. One critical responsibility of managers is to align the skills of human resources to the required tasks of the organization; therefore, it is not acceptable for managers to seek a low match with regard to matching skills to tasks. Additionally, only marginal or novice managers would be permitted the goal of achieving a random match; competent management should seek skill to task alignment beyond that achieved by a random match, continuously striving to attain a high skill to task match.

Overtraining is implied when frequent $\mathrm{KC}$ process participation (e6p in Tables 5 and 6 ) results in the worst organizational values among those of the three KC frequency options. However, this is largely a risk for certain short-term situations or with regard to some low skill to task match situations. These situations are only likely to occur when organizational tasks are unknown, when worker competence is unknown, or when knowledge workers select their own KC process independently of organizational goals. This suggests that frequent $\mathrm{KC}$ process participation resulting in overtraining is unlikely over time for all but low match conditions. There is a challenge to shortterm goals, however, where KC process participation should result in near-term high match alignment. If future tasks are intended to change dramatically rather than evolutionarily, the manager must plan carefully how such a transition will need to occur. Presuming that high knowledge depreciation and high task value represent an innovative market opportunity, particularly one such as bioinformatics, these results provide motivation for organizations to strive to align skills to tasks well.

The consideration of a sustained low match over time may imply either management strategy or management that is weak or naive. Under all conditions, one might consider the wisdom of remaining in a situation for which the organization does not clearly know how to compete. If this situation is true for a significant portion of a company, corporate strategy may need to be revisited. If it equates to one or a few departments, the situation may imply that the wrong workers are being hired or retained.

\section{KC Process Intensity}

We do not find support for hypothesis 2. To examine the effects of $\mathrm{KC}$ process intensity, we invoked Scenario II. In examining Table 6, we can see that an increase in $\mathrm{KC}$ intensity, in general, does not appear to substantially impact KC process decisions when the goal is the maximization of organizational value. In the event that KC process costs to $\mathrm{KC}$ process intensity are random, over the long term infrequent $\mathrm{KC}$ process participation appears to be preferred to frequent $\mathrm{KC}$ process participation for situations for all but shortterm situations or under high knowledge depreciation for the intermediate (24 time period) interval. For a low skill to task match, it would be expected that, under conditions of high or medium knowledge depreciation, no KC process participation is merited. However, it is interesting to note that under conditions of low knowledge depreciation, in all but the short term, infrequent KC process participation is preferred, but generally even frequent $K C$ process participation is preferred over no KC process participation. We cannot substantiate in this paper, but can speculate, that perhaps this is an effect of riding a market wave where anomalous, upward market trends benefit an overwhelming majority of suppliers. 
An interesting observation is the lack of negative effect on increasing intensity when achieving a high skill to task match. It would seem that the risk of overtraining with regard to the near and intermediate results and a high match is very low. In light of the dramatic difference in organizational value achieved by a high match (refer to Figure 2), these results encourage managers to err on the side of frequent $\mathrm{KC}$ processes.

\section{Knowledge Depreciation}

We find partial support for hypothesis 3 and note that the amount of knowledge depreciation affecting an organization is a major consideration in determining $\mathrm{KC}$ process strategy. The nature of the $\mathrm{KC}$ process decision under conditions of high knowledge depreciation is different from those under low to medium levels (refer to Table 5). With regard to high knowledge depreciation, the decision becomes largely binary: to maximize organizational profits, invest in KC processes (frequently for a high match or infrequently for a random match) or else consider no KC investment in the short term (as a tactical decision). For lower levels of knowledge depreciation, infrequent $\mathrm{KC}$ process participation is the predominant choice, with frequent $\mathrm{KC}$ process participation for obtaining a high match in the 12 and 24 time period options, and no KC processes preferred for the short term.

Under high knowledge depreciation conditions, it should be noted that many managers hesitate to engage knowledge workers in frequent $\mathrm{KC}$ process participation, fearing that workers may leave, wasting the investment for the organization, or that budget considerations must take precedence (Daniels 2003; Horner 2002; HR Focus 2004; Jusko and McClenahen 2004; McClenahen 2004; Poole 2004). Our results show that when managers invest in frequent $\mathrm{KC}$ processes to achieve a high match between the employees' skills and the tasks they need to achieve, a positive return can be expected even in the short term. It is only when it is unknown (on how to match the employees' skills to organizational tasks resulting in random or low matches) that $\mathrm{KC}$ process invest- ments need to be considered carefully. In this latter situation, short-term organizational benefits are negatively impacted by any participation in $\mathrm{KC}$ processes; however, infrequent $\mathrm{KC}$ process participation benefits the organization, in general, even in a random match situation over time. Actual high turnover would negatively influence our model due to the possibility that the skills of new hires are unknown, but previous research (Booth et al. 2002) has shown that training (a KC process) does reduce worker turnover.

\section{KC Process Selection}

Support for hypothesis 4 is conditional upon whether the organization's tasks are homogeneous or heterogeneous. In our model, the amount and type of $\mathrm{KC}$ process is primarily influenced by the gap between skills to anticipated task, and other factors such as knowledge depreciation and time. In one sense, these factors indirectly support the consideration of $\mathrm{KC}$ process as a bonus strategy: successful workers are likely to be given more responsibility thus requiring more knowledge and an understandable expectation for greater KC process participation. However, even this strategy overly simplifies the factors to consider for maximizing organizational benefits and group behavior.

In typical organizations, there are a variety of tasks requiring a combination of complementary and sometimes disparate skills. In general, the tasks cannot be expected to be homogeneous; therefore, the KC processes to help knowledge workers address their assigned tasks should not be expected to be homogeneous. So except for atypical organizations, we find that our results support hypothesis 4 .

\section{Longitudinal Effects of KC Processes}

In general, referring to Figure 2, we can see that a high skill to task alignment (i.e., match) greatly exceeds the value contribution to an organization over random and low match over time. While the range of organizational value in the short term is 
much smaller in the short term, the effects of correctly aligning knowledge workers' skills to their assigned tasks shows a dramatic increase over time.

We observe (from Table 5) that frequent KC process participation (every 6 months) provides the best organizational value when skills are highly matched to organizational tasks, supporting hypothesis 5. For all conditions of knowledge depreciation, if the match is low or random, the organization achieves a short-term benefit by providing no $\mathrm{KC}$ process but only by optimizing the short term to the detriment of longer-term considerations which also supports hypothesis 5 .

\section{Implications to Practice}

Workgroup composition and task alignment are significant contributors to causal ambiguity (Barney 1991), and optimal organizational value depends on a number of dynamic factors that affect these variables as is demonstrated by our model. Our simulation exercises the model to identify trends and valuation expectations within a specific organizational context. Our simulation results suggest to practitioners that task definition and ongoing $\mathrm{KC}$ process participation become more important as knowledge depreciation increases. It also advises practitioners to align the type of $\mathrm{KC}$ process to the requirements of the anticipated task. Finally, it reminds practitioners that reductions in $\mathrm{KC}$ process participation are likely to have negative longitudinal impacts on the organization.

Our model provides insights into complex decision making with regard to $\mathrm{KC}$ process investments. We demonstrate that task, worker competency, and knowledge depreciation are all relevant variables, in addition to measurable costs, in determining the optimal choice for organizational value. We suggest that managers should consider the generalized observations of this study rather than the specific values we employ. That is, it is better to consider decision points like "frequent" or "moderate" rather than specific values, such as 6 or 12 months, due to the context-specific nature of the knowledge worker organization.

\section{Implications to Research}

Knowledge creation is fundamental to the survival of businesses and yet it has not been extensively researched beyond organizational and behavioral theory. This study is significant to research as one of the first to address knowledge creation with perspectives from economics and information systems, in addition to organizational theory.

The model we propose decomposes into a number of complex variables, which are presented in a parsimonious manner, and addresses the dynamic nature of organizational knowledge creation and depreciation. Our model is driven by existing theory and extant research. The addition of a simulation prototype aids our ability to apply theoretical considerations rather than a mere simulation based entirely on random number generation. The latter instance implies data-driven development, whereas our approach is structured to assist theory-driven research that respects the contextual nature of knowledge.

In a complex setting and where research is at a new or embryonic stage, such as exists in considering knowledge creation valuation for knowledge workers, we consider simulation to be a reasonable and appropriate research method. Our model allows for the consideration of interaction between variables in a complex setting. Thus, it approximates the real world better than would an experimental method where only a few variables are considered. Additionally, it does not require negative effects that would occur in a real world setting to observe the same conditions. As an artificially controlled model, many results may occur as expected; however, the interesting results are those that do not exactly conform to expectations. Our results can be useful in guiding subsequent analysis, such as case study, survey, experimental, and quasi-experimental research addressing knowledge creation valuation.

Future research can address the nature of the process to help qualify details relating to $\mathrm{KC}$ process intensity. Our model assists in considering the aspects of who should participate in $\mathrm{KC}$ processes and how (i.e., intensity) they should participate. 
Future research is needed to address the nature of complementarity quantification. Our model does not directly address complementarity, such as the use of $\mathrm{KC}$ processes that change the nature of a task (or by removing certain task requirements that now may be performed by another in their task). Further contributions to decision criteria with regard to $\mathrm{KC}$ process and complementarity would benefit both researchers and practitioners.

Our model could be extended to consider different valuation levels of opportunity costs for each knowledge worker. In a real world setting, an organization may consider whether a certain $\mathrm{KC}$ process has primarily organizational (specific) or individual (general) value and such classification influences to what extent the organization will assume the costs. Similarly, even if it assumes the costs, workers are not always excited about participating in every applicable KC process. Issues relating to current schedules and conflicts relating to future task assignment can influence opportunity cost structure.

Our current model supports knowledge work that is aided by collaboration but not rigid specification. For instance, one would demand that one doctor who is fully qualified (e.g., 100 percent) perform neurosurgery, rather than allow five doctors who are all only 20 percent qualified to perform the surgery. Our model could be extended to allow for this type of rigid specification.

While our model supports almost all KC process participation over a do-nothing strategy, further research would be useful to consider the issues of task ambiguity with regard to excessive $\mathrm{KC}$ process participation (e.g., overtraining). Our current model assumes that anticipated tasks can be defined rationally. In the presence of task ambiguity, further research could consider decision criteria to minimize overtraining or, worse, undertraining scenarios. Future research could address individual perspectives to consider whether overtraining is negative (such as increasing turnover) or positive (such as increased innovation).

\section{Conclusion}

Our model provides a contribution, as one of the first to quantify the decision criteria required by managers and knowledge workers with regard to knowledge creation process investment decisions using organizational and economic theory. However, it becomes more interesting by providing insights not readily observed in common management practice with respect to these decisions, such as the disparity in short-term decisions (i.e., frequent $\mathrm{KC}$ process participation preferred for high match but no KC process participation for both random and low matches), the extent of the impact of high skill to task alignment over time as seen in Figure 2, and the lack of negative effect of increasing intensity for achieving high skill to task alignment. It is not uncommon for managers to leave KC process decisions predominantly to the discretion of individual workers. Under instances of high knowledge depreciation, however, it is unlikely that individual workers can optimize knowledge creation process decisions without organizational involvement in matching skills to task complexities. The organizational benefits of consistent and frequent knowledge creation process participation increase over time and as the match of skills to tasks improves.

In this study, we focus on the analytical and/or decision-making complexities for knowledge workers whose roles consist of significant amounts of knowledge work. The knowledge work is composed of a set of tasks, each of which requires a specific skill from a worker in order to accomplish the task satisfactorily. Frequent KC process participation, when it results in a high match of skills to task, provides significant organizational benefits.

Training may be considered a useful example of KC process participation, but knowledge creation does not require participation in formalized courses taught by external consultants. However, we do not consider ad hoc meetings or poorly managed gatherings to be considered adequate $\mathrm{KC}$ processes. The intent of $\mathrm{KC}$ process parti- 
cipation is that it is a formal process (i.e., with objectives, process, evaluation, and feedback) whereby external information or knowledge is exposed to knowledge workers in a systematic manner.

We are hesitant to recommend homogeneous $\mathrm{KC}$ participation. Sending everyone to the same KC process may need to be reconsidered unless both the individual skill sets are homogeneous and the task sets to be performed are homogenous. However, empirical research could be useful to examine the effect of homogeneous KC process participation on complementarity.

The ability of managers to productively utilize knowledge worker competency over time is a critical element of strategic management. It is important for managers to understand the relevant constructs affecting $\mathrm{KC}$ process investment decisions, the interaction effects of how these various constructs affect organizational outcomes, and how their own decisions can influence these outcomes.

\section{Acknowledgments}

The authors wish to sincerely thank the senior editor, Dr. Sambamurthy, the associate editor, and the anonymous reviewers for their invaluable comments. They have contributed significantly to strengthen this paper. The authors also thank $\mathrm{Dr}$. James Marsden, whose suggestions were gratefully appreciated.

\section{References}

Alavi, M., and Leidner, D. E. "Review: Knowledge Management and Knowledge Management Systems: Conceptual Foundations and Research Issues," MIS Quarterly (25:1), March 2001, pp. 107-136.

Argote, L., Beckman, S., and Epple, D. "The Persistence and Transfer of Learning in Industrial Settings," Management Science (36), 1990, pp. 117-127.
Anderson, J. R. Cognitive Psychology and its Implications ( $4^{\text {th }}$ ed.), W. H. Freeman and Company, New York, 1995.

Baland, J-M., and Francois, P. "Rent-Seeking and Resource Booms," Journal of Development Economics (61:2), April 2000, pp. 527-542.

Barney, J. "Firm Resources and Sustained Competitive Advantage," Journal of Management, (17:1), 1991, pp. 99-120.

Barua, A., Lee, S. C. H., and Whinston, A. B. "The Calculus of Reengineering," Information Systems Research (7:4), December 1996, pp. 409428.

Becker, G. S. "Investment in Human Capital: A Theoretical Analysis," Journal of Political Economy, October 1962, pp. 9-49.

Becker, G. S. The Economic Approach to Human Behavior, University of Chicago Press, Chicago, 1977.

Booth, A. L., Chen, Y-F., and Zoega, G. "Hiring and Firing: A Tale of Two Thresholds," Journal of Labor Economics (20:2), April 2002, pp. 217 248.

Bratley, P., Fox, B., and Schrge, L. A Guide to Simulation $\left(2^{\text {nd }}\right.$ ed. $)$, Springer-Verlag, New York, 1987.

Child, J., and Heavens, S. J. "The Social Constitution of Organizations and its Implications for Organizational Learning," Handbook of Organizational Learning and Knowledge, M. Dierkes, A. B. Antal, J. Child, and I. Nonaka (Eds.), Oxford University Press, Oxford, 2001, pp. 308326.

Christianse, E., and Venkatraman, N. "Beyond Sabre: An Empirical Test of Expertise Exploitation in Electronic Channels," MIS Quarterly (26:1), March 2002, pp. 15-38.

Coase, R. H. "The Nature of the Firm," Economica, (4), 1937, pp. 386-405.

Cohen, W. M., and Levinthal, D. A. "Absorptive Capacity: A New Perspective on Learning and Innovation," Administrative Science Quarterly (35), March 1990, pp.128-152.

Daniels, S. "Employee Training: A Strategic Approach to Better Return on Investment," The Journal of Business Strategy, (24:5), 2003, pp. 39-42.

Darr, E. D., Argote, L., and Epple, D. "The Acquisition, Transfer and Depreciation of Knowledge 
in Service Organizational Productivity in Franchises," Management Science (41:11), November 1995, pp. 1750-1613.

Davenport, T., and Grover, V. "Special Issue: Knowledge Management (Editorial)," Journal of Management Information Systems (18:1), 2001, pp. 3-4.

Debenham, J. "Knowledge Decay at a Normalised Database," in Database and Expert Systems Applications, $11^{\text {th }}$ International Conference, DEXA 2000, M. T. Ibrahim, J. Küng, and N. Revell (Eds.), Springer, London, 2000, pp.417426 (available online at http://linus.socs. uts.edu.au/ debenham/papers/DEXA-00.pdf).

Edgeworth, F. Y. Mathematical Psychics, Kegan Paul, London, 1881.

Fielden, M. "Data Validation," Industrial Management and Data Systems (90:4),1990, pp. 3-6.

Flamholtz, E. G., and Lacey, J. "The Implications of the Economic Theory of Human Capital for Personnel Management," Personnel Review (10:1), 1981, pp. 30-40.

Hahn, J., and Subramani, M. R. "A Framework of Knowledge Management Systems: Issues and Challenges for Theory and Practice," in Proceedings of the $21^{\text {st }}$ International Conference on Information Systems, W. J. Orlikowski, S. Ang, P. Weill, H. C. Krcmar, and J. I. DeGross (Eds.), Brisbane, Queensland, Australia, 2000, pp. 302-312.

Harrison, J. S., Hitt, M. A., Hoskisson, R. E., and Ireland, R. D. "Resource Complementarity in Business Combinations: Extending the Logic to Organizational Alliances," Journal of Management (27:6), 2001, pp. 679-690.

Hitt, M. A., Hoskisson, R. E., and Ireland, D. R. "Mergers and Acquisitions and Managerial Commitment to Innovation in M-Form Firms, Strategic Management Journal (11:5), Summer 1998, pp. 29-38.

Horner, S. M. "Training Budgets must Be Sacred Cows," National Underwriter (106:42), October 21, 2002, p. 18.

HR Focus. "Companies Cut Salaries, Hiring, Benefits to Pay for Health Care," (81:12), December 2004, p. 8.

Hubbard, A. "A Price Tag On Training," Mortgage Banking (55:5), February 1995, pp. 5-16.
Jensen, M. C., and Meckling, W. H. "Theory of the Firm: Managerial Behavior, Agency Costs, and Ownership Structure," Journal of Financial Economics (3:4), October 1976, pp. 305-360. Josefek Jr., R. A., and Kauffman, R. J. "Five Degrees of Separation: A Human Capital Model of Employment-Related Decision Making in the Information Technology Workforce," in Proceedings of the $32^{\text {nd }}$ Hawaii International Conference on Systems Sciences (HICSS-32), R. Sprague (Ed.), IEEE Computer Society Press, Los Alamitos, CA, 1999.

Jusko J., and McClenahen, J. S. "Dangerous Disconnect," Industry Week, (253:7), July 2004, pp. 26-32.

Laursen, K., and Foss, N. J. "New Human Resource Management Practices, Complementarities and the Impact on Innovation Performance," Cambridge Journal of Economics, (27), 2003, pp. 243-263.

Malcomson, J. M., Maw, J. W., and McCormick, B. "General Training by Firms, Apprentice Contracts, and Public Policy," European Economic Review, (47), 2003, pp. 197-227.

McClenahan, J. S. "The Numbers Deficit," Industry Week, July 1, 2004 (available online at http://www.industryweek.com/CurrentArticles/ Asp/articles.asp? Articleld=1629).

Milgrom, P., and Roberts, J. "Complementarities and Fit: Strategy, Structure, and Organizational Change in Manufacturing," Journal of ACcounting \& Economics (19:2-3), 1995, pp. 179209.

Mincer, J. "On the Job Training: Costs, Returns and Some Implication," The Journal of Political Economy (70:5), 1962, pp. 50-79.

Ndlela, L. T., and du Toit, A. S. A. "Establishing A Knowledge Management Programme For Competitive Advantage in an Enterprise," International Journal of Information Management (21:2), April 2001, pp. 151-165.

Nonaka, I. "A Dynamic Theory of Organizational Knowledge Creation," Organizational Science (5:1), February 1994, pp. 14-37.

Nonaka, I. "The Knowledge-Creating Company," Harvard Business Review (69:6), 1991, pp. 96103.

Nonaka, I., and Tackeuchi, H. The KnowledgeCreating Company, Oxford Press, New York, 1995. 
Pawlowsky, P. "The Treatment of Organizational Learning in Management Science," Handbook of Organizational Learning and Knowledge, M. Dierkes, A. B. Antal, J. Child, and I. Nonaka (Eds.), , Oxford University Press, Oxford, 2001, pp. 89-117.

Piaget, J. Science of Education and the Psychology of the Child, Viking Press, New York, 1969.

Poole, D. "Cutting Training Corners Leaves No Future Talent," Precision Marketing, February 27, 2004, p. 14.

Prat, A. "Should a Team Be Homogeneous?," European Economic Review (46:7), 2002, pp. 1187-1207.

Purvis, R. L., Sambamurthy, V., and Zmud, R. W. "The Assimilation of Knowledge Platforms in Organizations: An Empirical Investigation," Organization Science (12:2), 2001, pp. 117-135.

Schacter, D. L. The Seven Sins of Memory: How the Mind Forgets and Remembers, Houghton Mifflin, Boston, 2001.

Shaw, W. D. "Searching for the Opportunity Cost of an Individual's Time," Land Economics (68:1), February 1992, pp.107-115.

Schilling, M. A., Vidal, P., Ployhart, R. E., and Marangoni, A. "Learning by Doing Something Else: Variation, Relatedness, and the Learning Curve," Management Science (49:1), January 2003, pp. 39-56.

Simon, H. "A Formal Theory of the Employment Relationship," Econometrica, (19:3), 1951, pp. 293-305.

St. John, C. H., and Harrison, J. S. "Manufacturing-Based Relatedness, Synergy, and Coordination," Strategic Management Journal (20:2), February 1999, pp. 129-146.

Stevens, M. "An Investment Model for the Supply of Training by Employers," The Economic Journal (104:424), May 1994, pp. 556-571.

Teece, D. J. "Economic Analysis and Strategic Management," California Management Review (26), Spring 1984, pp. 87-110.

Vera-Munoz, S. C. "The Effects of Accounting Knowledge and Context on the Omission of Opportunity Costs in Resource Allocation Decisions," The Accounting Review (73:1), January 1998, pp. 47-72.

Wade, M., and Hulland, J. "Review: The Resource-Based View and Information Systems
Research: Review, Extension, and Suggestions for Future Research," MIS Quarterly (28:1), March 2004, pp. 107-142.

Wenger, E. C., and Snyder, W. M. "Communities of Practice," Harvard Business Review on Organizational Learning, Harvard Business School Press, Boston, 2001, pp. 1-20.

Williamson, O. E. "The Organization of Work," Journal of Economic Behavior and Organization (1:1), 1980, pp. 5-38.

Zell, D. "Overcoming Barriers to Work Innovations: Lessons Learned at Hewlett-Packard," Organizational Dynamics (30:1), Summer 2001, pp. 77-86.

\section{About the Authors}

Andrew Chen received his Ph.D. in Operations and Information Management from University of Connecticut. His current teaching and research interests include knowledge management, innovation adoption, electronic commerce strategy, database management, and business and Web applications. His research has appeared in Decision Support Systems, European Journal of Operational Research, Journal of Electronic Commerce Research, Journal of Management Information Systems, MIS Quarterly, and international conferences.

Theresa Edgington is an Ph.D. candidate (ABD) in Information Systems at Arizona State University and a charter CABIT researcher. She worked in the IT industry for over 20 years spanning technical to director-level roles relating to technology innovation involving e-business, artificial intelligence, advanced databases, reengineering, transaction processing and messaging, and application development. She is a former OMG member and has presented at numerous industry conferences. Her research interests include knowledge creation and innovation, ontology and knowledge representation, failure analysis, strategic management, systems analysis, and computer-supported collaboration. Her research has appeared in Communications of the ACM, MIS Quarterly, and at academic conferences. 